Journal of Applied Fluid Mechanics, Vol. 2, No. 1, pp. 71-96, 2009.

Available online at www.jafmonline.net, ISSN 1735-3572, EISSN 1735-3645.

DOI: $10.36884 / \mathrm{jafm} \cdot 2.01 .11858$

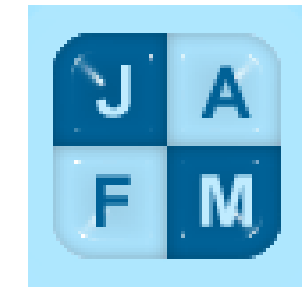

\title{
A Generalized Lattice Boltzmann Method for Three-Dimensional Incompressible Fluid Flow Simulation
}

\author{
A. R. Rahmati ${ }^{1}$ and M. Ashrafizaadeh ${ }^{2}$ \\ 1, 2 Department of Mechanical Engineering, Isfahan University of Technology, Isfahan, 8415683111, Iran. \\ E-mail:rahmati@me.iut.ac.ir
}

(Received September 15, 2007; accepted October 29, 2008)

\begin{abstract}
In this work, a 19-bit Incompressible Generalized Lattice Boltzmann (IGLB) method has been proposed for threedimensional incompressible fluid flow simulation, for the first time. Equilibrium moments in moment space are derived from an incompressible BGKLB method. The incompressible Navier-Stokes equations can be recovered through the Chapman-Enskog multi-scale expansion without artificial compressible effects. To compare the performance of proposed model, several benchmark problems (such as a cubic lid-driven cavity flow, flow over a backward-facing step, and a double shear flow) are solved and the results are compared with those of both 19-bit Incompressible BGK Lattice Boltzmann (IBGKLB) method and existing CFD simulations. It is shown that the stability and accuracy of the 19-bit IGLB method is better than those of the 19-bit IBGKLB method; in fact with the IGLB model we can increase the Reynolds number by factor of 2.5 and still get stable results. The proposed 3-D IGLB method is successfully expanded and applied to simulation of the 3-D incompressible buoyancy driven flows. The results of the 3-D steady-state natural convection in an air-filled differentially heated cubic cavity obtained by the extended model comply well with the existing data in literature. In addition, natural convection from a discrete heat source which is mounted flush with the bottom wall of a horizontal enclosure is simulated. The obtained results indicate that the proposed method is very convenient for simulation of thermally driven flow problems.
\end{abstract}

Keywords: BGK lattice Boltzmann method, Generalized LBM, Hybrid thermal lattice Boltzmann method, D3Q19

\section{NOMENCLATURE}

B

$c$

$c_{s}$

D

$\mathbf{e}_{i}$

e

F

$f_{i}$

$f_{i}^{e q}$

$g$

$H$

$\mathbf{M}$

$M$

$m$

$p$

Pr total number of lattice streaming vectors

magnitude of lattice streaming

vectors

speed of sound

dimension of system

lattice streaming vector in $i$

direction

kinetic energy

external force

density distribution function

equilibrium density distribution

function

acceleration due to gravity

height

moment space

transformation matrix

moment

pressure

$u / a$, Prandtl number q

$R a$

$U$

$U_{0}$

$u, v, w$

V

$\mathbf{u}$

$\hat{S}$

$S$

$t$

$T$

$x, y, z$

Greek symbols

a

$b$

$\delta r$

$\delta t$ heat flux vector position vector $\frac{g b \mathrm{D} T H^{3}}{u a}$, Rayleigh number maximum velocity at the inlet top lid velocity

$\mathrm{x}, \mathrm{y}$ and $\mathrm{z}$ components of flow velocity vector discrete velocity space flow velocity vector relaxation matrix step height time temperature Cartesian coordinate system

fluid thermal diffusivity thermal expansion coefficient lattice spacing time step 
A. R. Rahmati and M. Ashrafizaadeh / JAFM, Vol. 2, No. 1, pp. 71-96, 2009.

$\begin{array}{ll}\varepsilon & \text { square of kinetic energy } \\ \omega_{\varepsilon}, \omega_{\varepsilon j}, \omega_{x x} & \text { free parameters } \\ \rho & \text { local density } \\ \tau & \text { local relaxation time }\end{array}$

\section{INTRODUCTION}

The Lattice Boltzman Method (LBM) (McNamara and Zanetti. 1988; Chen and Doolen 1998), a derivative of lattice gas automaton method (Wolfram 1986; Frisch et al. 1986; Higuera and Jimenez 1989), is a relatively new numerical approach for fluid flow simulation. Due to its micro and meso scale theoretical bases as well as its ease of numerical implementation, it has gained a fast growing popularity in science and engineering, particularly for complex fluid flows (Shan and Chen. 1993; Ladd 1994; Chen et al. 1995; Boon et al. 1996; Pan et al. 2004; Zheng et al. 2006; Rahmati and Ashrafizaadeh 2007; Rahmati et al. 2008a,b,c,d,e,f,g).

Perhaps, the simplest and consequently the most popular form of the LBM is the BGK Lattice Boltzmann method (BGKLB) (Qian et al. 1992; Chen et al. 1992). However, this simplicity comes at the expense of some deficiencies (e.g. numerical instability and inaccuracy in implementing boundary conditions). To overcome some of the BGKLB deficiencies, the Generatlized Lattice Boltzmann (GLB) method (D'Humières 1992) has been developed.

Most of the existing GLB models have been constructed for the compressible Navier-Stokes equations in the low Mach number limit. As a result, the recovered macroscopic equations through the Chapman-Enskog multi-scale expansion are the nearly incompressible Navier-Stokes equations. Therefore, some compressibility errors can exist when incompressible flows are simulated. In this paper, we present some results to demonstrate compressible errors of a BGKLB model in comparison with those of an incompressible BGKLB (IBGKLB) model. On the other hand, the incompressible Navier-Stokes equations are of great importance in theory and application. Hence, it is necessary to develop exact incompressible LB models.

Du et al. (2006) showed that IGLB models are more accurate than IBGKLB models using a two-dimensional lid-driven cavity simulation. Following the method of GLB model, a three- dimensional incompressible GLB (IGLB) model is proposed in the present work. In this model the equilibrium moments in moment space are derived from an earlier incompressible BGK method by Guo et al. (2000). The Navier-Stokes equations can be recovered through the Chapman-Enskog multi-scale expansion without artificial compressibility effects. To compare the performance of the new IGLB model with that of the IBGKLB model, numerical models have been developed using a D3Q19 lattice. The numerical stability and performance of these models are investigated through several well-known fluid flow benchmarks including a three-dimensional lid-driven cavity flow, flow over a backward-facing step, and a double shear flow.

\author{
$v \quad$ kinematic viscosity \\ Subscripts and superscripts \\ eq equilibrium state \\ $i \quad$ lattice streaming vector direction
}

The proposed 3-D IGLB method is successfully extended and utilized in simulation of the three-dimensional incompressible buoyancy induced flows. Natural convection in an air-filled side-heated cubic cavity and natural convection from a heat source mounted on the bottom wall of a horizontal enclosure are simulated by the extended model.

The rest of this paper is organized as follows. Section two describes governing equations associated with both the incompressible BGK lattice Boltzmann and the incompressible generalized lattice Boltzmann methods. Section three explains the hybrid thermal lattice Boltzmann method. Results and Discussions are presented in section four and followed by conclusion in the last section.

\section{GOVERNING EQUATIONS}

\subsection{Incompressible BGK Lattice Boltzmann Method}

The LBM utilizes a particle distribution function to describe the collective behavior of fluid molecules. In LB methods, particles are assumed to move synchronously along the bonds of a regular lattice, and satisfy the discrete form of the lattice Boltzmann equation. Basically, this method consists of the following two phases:

(i) Streaming: in this phase, particles move along lattice bonds to the neighboring lattice nodes.

(ii) Collision: in this phase, particles on the same lattice node shuffle their velocities locally such that mass and momentum are conserved.

One of the most widely used collision model is the Bhatnagar-Gross-Krook (BGK) collision operator (Bhatnagar et al. 1954), which applies the single time relaxation approximation. The collision step occurs locally in the sense that it does not require any spatial and temporal derivatives. The streaming step following the collision moves the updated distribution functions to neighboring nodes by perfect shift on a uniform lattice.

For flow field, the evolution equation using the density distribution function, $f_{i}$, and the collision operator $\Omega_{i}(f)$ is given as the following form (Hou 1995):

$f_{i}\left(\mathbf{r}+\mathbf{e}_{i} \delta t, t_{n}+\delta t\right)-f_{i}\left(\mathbf{r}, t_{n}\right)=\Omega_{i}(f)$,

here $\mathbf{r}$ is the position of each lattice node, $t_{n}$ is the discrete time, $\delta t$ is the time step, and $\mathbf{e}_{i}$ is the particle speed in the $i$ direction. A simple model for collision operator $\Omega_{i}(f)$ is BGK model (Bhatnagar et al. 1954) given by: 
A. R. Rahmati and M. Ashrafizaadeh / JAFM, Vol. 2, No. 1, pp. 71-96, 2009.

$\Omega_{i}(f)=-\frac{f_{i}\left(\mathbf{r}, t_{n}\right)-f_{i}^{e q}\left(\mathbf{r}, t_{n}\right)}{\tau}$,

where $t$ is the relaxation time and $f_{i}^{e q}$ is the equilibrium density distribution function.

Therefore, the BGK lattice Boltzmann equation can be written as (Hou 1995; He and Luo 1997):

$$
\begin{aligned}
f_{i}\left(\mathbf{r}+\mathbf{e}_{i} \delta t, t_{n}+\delta t\right)= & f_{i}\left(\mathbf{r}, t_{n}\right) \\
& -\frac{f_{i}\left(\mathbf{r}, t_{n}\right)-f_{i}^{e q}\left(\mathbf{r}, t_{n}\right)}{\tau},
\end{aligned}
$$

For D3Q19 model (c.f. Fig. 1), the lattice velocities are defined as:

$$
\mathbf{e}_{i}=\left\{\begin{array}{lc}
0, & i=0, \\
( \pm 1,0,0) c,(0, \pm 1,0) c,(0,0, \pm 1) c, & i=1 \text { to } \quad 6, \\
( \pm 1, \pm 1,0) c,( \pm 1,0, \pm 1) c,(0, \pm 1, \pm 1) c, & i=7 \text { to } \quad 18
\end{array}\right.
$$

where $c$ is the particle velocity and is given by $\delta x / \delta t$. In lattice units, the time step $\delta t$ is set equal to 1 , as is the lattice spacing $(\delta x=\delta t=1)$.

For Incompressible BGKLB model which is proposed by Guo et al. (2000), the equilibrium functions for the density distribution function are given as:

$$
f_{i}^{e q}=\left\{\begin{array}{c}
\rho_{0}-\left(1-w_{0}\right) \frac{p}{c_{s}^{2}}+s_{0}(\mathbf{u}), \quad i=0, \\
w_{i} \frac{p}{c_{s}^{2}}+s_{i}(\mathbf{u}), \quad i=1 \text { to } 18,
\end{array}\right.
$$

where $w_{0}=\frac{1}{3}, w_{i}=\frac{1}{18}$ for $i=1$ to 6 and $w_{i}=\frac{1}{36}$ for $i$ $=7$ to 18 . Also $s_{0}(\mathbf{u})$ and $s_{i}(\mathbf{u})$ are given by:

$$
s_{0}(\mathbf{u})=-\frac{|\mathbf{u}|^{2}}{2 c_{s}^{2}} w_{0},
$$

$s_{i}(\mathbf{u})=w_{i}\left[3 \mathbf{e}_{i} \cdot \mathbf{u}+4.5\left(\mathbf{e}_{i} \cdot \mathbf{u}\right)^{2}-1.5|\mathbf{u}|^{2}\right]$.

In addition, $\rho_{0}, \mathbf{u}$ and $p$ are calculated by:

$$
\begin{aligned}
& \rho_{0}=\sum_{i=0}^{b} f_{i}, \\
& \mathbf{u}=\sum_{i=1}^{b} c \mathbf{e}_{i} f_{i},
\end{aligned}
$$

$$
p=\frac{c_{s}^{2}}{1-w_{0}}\left[\sum_{i=1}^{b} f_{i}+S_{0}(\mathbf{u})\right]
$$

where $b$ is the number of directions in the lattice model. Furthermore, the sound speed is $c_{s}=c / \sqrt{3}$.

In order to derive the incompressible Navier-Stokes equations, $\rho_{0}$ should be a fixed quantity, for example, $\rho_{0}$ is equal with 0 (Guo et al. 2000).

The incompressible Navier-Stokes equations can be derived from the IBGKLB model using a ChapmannEnzkog multi-scale expansion. From the expansion, these equations are given by the following expressions:

$\nabla \cdot \mathbf{u}=0$

$\frac{\partial(\mathbf{u})}{\partial t}+\nabla \cdot(\mathbf{u u})=-\nabla p+v\left[\nabla^{2}(\mathbf{u})\right]$

where kinematic viscosity is calculated by :

$v=(2 \tau-1) / 6$.

The IBGKLB model has some deficiencies (Lallemand and Luo 2000). One of these deficiencies is the numerical instability, and as soon as the relaxation time approaches 0.5 , this model may lead to numerical instability. In order to alleviate this shortcoming of the IBGKLB model, the generalized lattice Boltzmann method can be used.

\subsection{Incompressible Generalized Lattice Boltzmann Method}

For a GLB model with B $(=b+1)$ discrete velocities $\left\{\mathbf{e}_{i} \mid i=0,1, \ldots, b\right\}$, a set of density distribution functions $\left\{f_{i}\left(\mathbf{r}, t_{n}\right) \mid i=0, \ldots, b\right\}$ is defined on each lattice node $\mathbf{r}$. The collision step is executed in the moment space $\mathbf{M}$, while the streaming step is performed in the velocity space $V$. The evolution equation for the GLB on a D-dimensional lattice with discrete time $t_{n}$ can be written as (D'Humières et al. 2002):

$$
\begin{array}{r}
\left|f_{i}\left(\mathbf{r}+e_{i} \delta t, t_{n}+\delta t\right)\right\rangle-\left|f_{i}\left(\mathbf{r}, t_{n}\right)\right\rangle=\Omega\left(\mathbf{r}, t_{n}\right) \\
=M^{-1} S\left[\left|m_{i}\left(\mathbf{r}, t_{n}\right)\right\rangle-\left|m_{i}^{(e q)}\left(\mathbf{r}, t_{n}\right)\right\rangle\right],
\end{array}
$$

where symbol |.> denotes a column vector. Therefore

$$
\begin{aligned}
&\left|f_{i}\left(\mathbf{r}+\mathbf{e}_{i} \delta t, t_{n}+\delta t\right)\right\rangle= \\
&\left(\begin{array}{rr}
f_{0}\left(\mathbf{r}, t_{n}+\delta t\right) & f_{1}\left(\mathbf{r}+\mathbf{e}_{1} \delta t, t_{n}+\delta t\right) \\
\ldots & f_{b}\left(\mathbf{r}+\mathbf{e}_{b} \delta t, t_{n}+\delta t\right)
\end{array}\right)^{T},
\end{aligned}
$$


A. R. Rahmati and M. Ashrafizaadeh / JAFM, Vol. 2, No. 1, pp. 71-96, 2009.

$$
\begin{aligned}
& \left|f_{i}\left(\mathbf{r}, t_{n}\right)\right\rangle=\left(\begin{array}{llll}
f_{0}\left(\mathbf{r}, t_{n}\right) & f_{1}\left(\mathbf{r}, t_{n}\right) & \ldots & \left.f_{b}\left(\mathbf{r}, t_{n}\right)\right)^{T},
\end{array}\right. \\
& \left|m_{i}\left(\mathbf{r}, t_{n}\right)\right\rangle \equiv\left(m_{0}\left(\mathbf{r}, t_{n}\right), m_{2}\left(\mathbf{r}, t_{n}\right), \ldots, m_{b}\left(\mathbf{r}, t_{n}\right)\right)^{T}, \\
& \left|m_{i}^{e q}\left(\mathbf{r}, t_{n}\right)\right\rangle \equiv\left(m_{0}^{e q}\left(\mathbf{r}, t_{n}\right), m_{1}^{e q}\left(\mathbf{r}, t_{n}\right), \ldots, m_{b}^{e q}\left(\mathbf{r}, t_{n}\right)\right)^{T}
\end{aligned}
$$

where $\mathrm{T}$ is the transpose operator and $m_{i}$ is moment in the $i$ direction and $m_{i}^{e q}$ is its corresponding equilibrium state. $M$ is a $B \times B$ matrix which linearly transforms the distribution functions $|f\rangle \in V$ to the moments $|m\rangle \in \mathbf{M}$ and vice versa the moments $|m\rangle \in \mathbf{M}$ to the distribution functions $|f\rangle \in V$, i.e.

$$
|m\rangle=M|f\rangle, \text { and } \quad|f\rangle=M^{-1}|m\rangle .
$$

The transformation matrix $M$ is constructed from the monomials of the discrete velocity components $e_{i \alpha}^{m} e_{j \beta}^{n} \ldots e_{k \gamma}^{l}$, where $\alpha, \beta$ and $\gamma \in\{x, y, z\}$, by means of the Gram-Schmidt orthogonalization procedure (Lallemand and Luo 2000, 2003, Bouzidi et al. 2001). The row vectors of $M$ are mutually orthogonal, that is, $M M^{T}$ is a diagonal matrix, but not normalized, so all the elements of matrix $M$ are integers.

The transformation matrix $M$ for the nineteen velocity model in three dimensions (D3Q19) is given as (D’Humières et al. 2002):

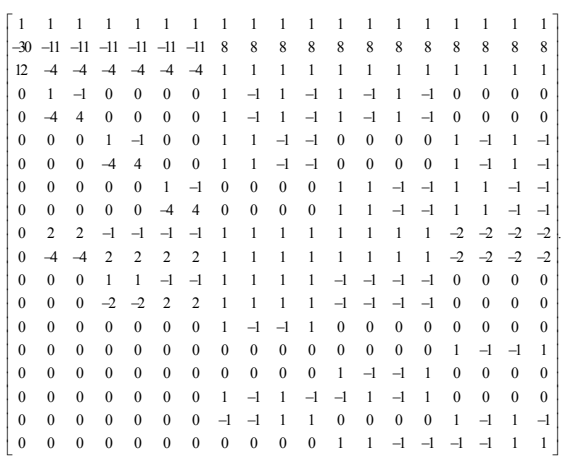

The nineteen moments for the nineteen-velocity model in three dimensions (D3Q19) are:

$$
|m\rangle=\left(\begin{array}{cccccccccccc}
\rho_{0} & e & \varepsilon & u_{x} & q_{x} & u_{y} & q_{y} & u_{z} & q_{z} & 3 p_{x x} & & \\
& & 3 \pi_{x x} & p_{w w} & \pi_{w w} & p_{x y} & p_{y z} & p_{x z} & m_{x} & m_{y} & m_{z}
\end{array}\right)^{T},
$$

where $m_{0}=\rho_{0}$ is the mass density, $m_{1}=e$ is the energy, $m_{2}=\varepsilon$ is the square of energy, $m_{3,5,7}=u_{x, y, z}$ are the components of the velocity vector $\mathbf{u}=\left(u_{x}, u_{y}, u_{z}\right)$, $m_{4,6,8}=q_{x, y, z}$ are components of the heat flux $\mathbf{q}=\left(q_{x}, q_{y}, q_{z}\right), \quad m_{9}=3 p_{x x} \quad, \quad m_{11}=p_{w w} \quad$ and $m_{3,14,15}=p_{x y, y z, z x}$ are components of the traceless and symmetric strain-rate tensor, $m_{10}=3 \pi_{x x}$ and $m_{12}=\pi_{w w}$ are the fourth-order moments, $m_{16,17,18}=m_{x, y, z}$ are the thirdorder moments. Among these moments, the mass density $\rho_{0}$ and the velocity vector $\mathbf{u}$ are invariant quantities and the rest of them are non-invariant quantities.

The equilibria of the moments, $m^{(e q)}$, are the functions of the invariant moments, which are the mass density $\rho_{0}$ and the velocity vector $\mathbf{u}$ for athermal fluids. However, in the current method, the pressure $p$ has been used instead of the mass density $\rho_{0}$, i.e., $m^{(e q)}\left(\mathbf{r}, t_{n}\right)=$ $m^{(e q)}\left(p\left(\mathbf{r}, t_{n}\right), u\left(\mathbf{r}, t_{n}\right)\right)$.

For the D3Q19 model, the equilibria for the non-conserved moments are given by the following relations (D'Humières et al. 2002):

$$
\begin{aligned}
& e^{(e q)}=\gamma_{1} p+19\left(u_{x}^{2}+u_{y}^{2}+u_{z}^{2}\right), \\
& \varepsilon^{(e q)}=\gamma_{2} p+\frac{11}{2}\left(u_{x}^{2}+u_{y}^{2}+u_{z}^{2}\right), \\
& q_{x}^{(e q)}=\frac{-2}{3} u_{x}, \\
& q_{y}^{(e q)}=\frac{-2}{3} u_{y}, \\
& q_{z}^{(e q)}=\frac{-2}{3} u_{z}, \\
& p_{x x}^{(e q)}=u_{x}^{2}-\frac{1}{3}\left(u_{x}^{2}+u_{y}^{2}+u_{z}^{2}\right), \\
& p_{w w}^{(e q)}=u_{y}^{2}-u_{z}^{2}, \\
& p_{x y}^{(e q)}=u_{x} u_{y}, \\
& p_{y z}^{(e q)}=u_{y} u_{z}, \\
& p_{x z}^{(e q)}=u_{x} u_{z}, \\
& \pi_{x x}^{(e q)}=\frac{-1}{2} p_{x x}^{(e q)}, \\
& \pi_{w w}^{(e q)}=\frac{-1}{2} p_{w w}^{(e q)}, \\
& m_{x}^{(e q)}=m_{y}^{(e q)}=m_{z}^{(e q)}=0 .
\end{aligned}
$$

In the present work, $\gamma_{1}$ and $\gamma_{2}$ are calculated so that the incompressible Navier-Stokes equations can be derived correctly. These quantities are as follows:

$$
\begin{aligned}
& \gamma_{1}=57, \\
& \gamma_{2}=-27
\end{aligned}
$$

The relaxation matrix $\hat{S}$ is diagonal in the moment space $\mathbf{M}$ :

$$
\hat{S} \equiv \operatorname{diag}\left(\begin{array}{llllllllllllll}
1 & s_{1} & s_{2} & 1 & s_{4} & 1 & s_{4} & 1 & s_{4} & s_{9} & & & \\
& & & s_{10} & s_{9} & s_{10} & s_{13} & s_{13} & s_{13} & s_{16} & s_{16} & s_{16}
\end{array}\right) .
$$


A. R. Rahmati and M. Ashrafizaadeh / JAFM, Vol. 2, No. 1, pp. 71-96, 2009.

The speed of sound in the model is $c_{s}=c / \sqrt{3}$.

The incompressible Navier- stokes equations can be derived from this method using a Chapmann-Enzkog multi-scale expansion. More details are presented in Ref. (Du et al. 2006). From this expansion, the kinematic viscosity $v$ is given as:

$v=\frac{1}{3}\left(\frac{1}{s_{9}}-\frac{1}{2}\right)=\frac{1}{3}\left(\frac{1}{s_{13}}-\frac{1}{2}\right)$.

It should be noted that the present model reduces to the IBGKLB model if we use a single relaxation parameter for all the moments, i.e. $s_{i}=1 / \tau$. In the next section, the thermal lattice Boltzmann methods are explained.

\section{HYBRID THERMAL LATTICE BOLTZMANN METHOD}

Although the kinetic-based lattice Boltzmann method has attained considerable success in simulating fluid flows and modeling the physics in fluids (Rahmati and Ashrafizaadeh 2007; Rahmati et al. 2008a,b,c,d,e,f,g). However, the application of lattice Boltzmann model to thermal problems has not achieved great prosperity for the thermal models due to the severe numerical instability caused by breaking the isothermal condition (Lallemand and Luo 2003).

Constructing LB models for thermal flows remains challenging in the LBM community, although some efforts have been made from various viewpoints. A recent comprehensive review on this topic can be found elsewhere (Lallemand and Luo 2003a,b).

The existing approaches for thermal LB models can be categorized into three categories, i.e., the multi-speed lattice Boltzmann scheme (Teixeira et al. 2000), the double-density-distribution-function lattice Boltzmann (DDDFLB) approach (Chen and Doolen 1998; Peng et al. 2003), and the hybrid thermal lattice Boltzmann (HTLB) technique (Lallemand and Luo 2003a,b; van Treeck et al 2006; Mezrhab et al. 2004). The multi-speed scheme is a straightforward extension of the isothermal LB models, in which only the density distribution function is used; the DDDFLB approach uses two different density distribution functions, one for the velocity field and the other for the internal energy field; and the HTLB technique is similar to the DDDFLB approach except that the internal energy equation is solved by finite-difference methods, rather than by solving the LB model (Lallemand and Luo 2003a,b).

In the present study, the proposed IGLB method is extended based on HTLB technique (Lallemand and Luo 2003a,b; van Treeck et al. 2006) for simulation of thermal flows and successfully applied to simulate natural convection in a differentially heated cubic cavity and to simulate natural convection from a discrete heat source on the bottom of a horizontal enclosure.
The equation used for solving the temperature field is given by:

$$
\begin{aligned}
\frac{\partial T}{\partial t}+u \frac{\partial T}{\partial x}+v & \frac{\partial T}{\partial y}+w \frac{\partial T}{\partial z}= \\
& \alpha\left(\frac{\partial^{2} T}{\partial x^{2}}+\frac{\partial^{2} T}{\partial y^{2}}+\frac{\partial^{2} T}{\partial z^{2}}\right),
\end{aligned}
$$

where $\alpha$ is the fluid thermal diffusivity. The energy equation is discretized by the finite-difference technique. In this scheme, $\mathrm{x}, \mathrm{y}$ and $\mathrm{z}$ components of heat flux and Laplassian operator can be discretized as the following formulae (Lalleman and Lou, 2003b):

$$
\begin{aligned}
& \frac{\partial T(x, y, z)}{\partial x}=T(x+1, y, z)-T(x-1, y, z)- \\
& \frac{1}{8}[T(x+1, y+1, z)-T(x-1, y+1, z)+ \\
& T(x+1, y-1, z)-T(x-1, y-1, z)+ \\
& T(x+1, y, z+1)-T(x-1, y, z+1)+ \\
& T(x+1, y, z-1)-T(x-1, y, z-1)] \text {, } \\
& \frac{\partial T(x, y, z)}{\partial y}=T(x, y+1, z)-T(x, y-1, z)- \\
& \frac{1}{8}[T(x+1, y+1, z)-T(x+1, y-1, z)+ \\
& T(x-1, y+1, z)-T(x-1, y-1, z)+ \\
& T(x, y+1, z+1)-T(x, y-1, z+1)+ \\
& T(x, y+1, z-1)-T(x, y-1, z-1)], \\
& \frac{\partial T(x, y, z)}{\partial z}=T(x, y, z+1)-T(x, y, z-1)- \\
& \frac{1}{8}[T(x+1, y, z+1)-T(x+1, y, z-1)+ \\
& T(x-1, y, z+1)-T(x-1, y, z-1)+ \\
& T(x, y+1, z+1)-T(x, y+1, z-1)+ \\
& T(x, y-1, z+1)-T(x, y-1, z-1)] \text {, } \\
& \left(\frac{\partial^{2} T(x, y, z)}{\partial x^{2}}+\frac{\partial^{2} T(x, y, z)}{\partial y^{2}}+\frac{\partial^{2} T(x, y, z)}{\partial z^{2}}\right)= \\
& 2[T(x+1, y, z)+T(x-1, y, z)+T(x, y+1, z)+ \\
& T(x, y-1, z)+T(x, y, z+1)+T(x, y, z-1)]- \\
& \frac{1}{4}[T(x+1, y+1, z)+T(x-1, y+1, z)+ \\
& T(x+1, y-1, z)+T(x-1, y-1, z)+ \\
& T(x, y+1, z+1)+T(x, y-1, z+1)+ \\
& T(x, y+1, z-1)+T(x, y-1, z-1)+ \\
& T(x+1, y, z+1)+T(x-1, y, z+1)+ \\
& T(x+1, y, z-1)+T(x-1, y, z-1)]-9 T(x, y) .
\end{aligned}
$$

It should be noted that the stencil used for finite difference operator must have the same symmetries as those of the discrete velocity set of the model, i.e. it is a nineteen-point stencil for the D3Q19 model. This stencil helps to improve the numerical stability of the scheme. 
The lattice Boltzmann equation may include external forces such as gravity force and buoyancy force. For a force term $F$, one can add it to the momentum. In order to conserve mass up to a second order one in ChapmanEnskog analysis, the net effect of force term $F$ is such that the resultant momentum is equal to $V+F d t / 2$. Preferably, it is better to add one-half of the force term before the relaxation, and one-half after it.

\section{Results}

\subsection{Description of the Errors Due to Compressibility}

In order to illustrate the error due to compressibility, a cubic cavity flow (c.f. Fig. 2) is simulated by both compressible BGKLB (CBGKLB) and incompressible BGKLB (IBGKLB) models. In these simulations, an $81 \times 81 \times 81$ lattice is used. In order to investigate the compressibility error, velocity at the top edge of the cavity is varied. The Reynolds number is about 400 .

Tables 1 and 2 show both maximum and minimum values of the $\mathrm{x}$ and $\mathrm{z}$ components of velocity and those of $\mathrm{Ku}$ et al. (1987). As shown in the tables, the results of the incompressible model are more compatible with $\mathrm{Ku}$ 's results (Ku et al. 1987) compared to those of the compressible model.

In the case of the compressible model, it is seen that the accuracy of the results deteriorates by increasing the velocity at the top plate of the cavity. While the flow field is simulated by an incompressible lattice Boltzmann method, the compressibility error is removed and it is possible to increase the velocity at the top wall of the cavity for the simulation of the flow field in higher Reynolds number.

In the next section, the three-dimensional lid-driven cavity flow, three-dimensional flow over backward-facing step, and three-dimensional double shear flow problems are simulated by the proposed model, i.e., IGLB model, and the obtained results are compared to the IBGKLB results and the existing CFD numerical data.

\subsection{Simulation of Isothermal Fluid Flows}

\subsubsection{Cubic Cavity Flow}

Figure 2 shows a schematic view of the three-dimensional cavity geometry and boundary conditions used for the simulation. The model problem consists of a cubic cavity with a constant velocity moving top lid. The cavity is filled with an incompressible viscous fluid. This test case is a classic benchmark for which many simulation results exist in the literature. In this simulation, an 81-cubic lattice is used.

Initially a constant pressure $p=1 / 3$ is prescribed for the whole cavity field, and the velocities in the interior of the cavity are set to zero. On the top face, the $\mathrm{x}$ component of velocity is $U_{0}$, which is set to 0.1 , and the $y$ and $z$ components of velocity are zero. The no-slip boundary condition is imposed on other walls. The results are presented for two Reynolds numbers (400 and 1000) and compared with those of $\mathrm{Ku}$ et al. (1987).

To investigate the performance of the IGLB model and compare its stability with that of an IBGKLB model, the three-dimensional lid-driven cavity flow has been simulated using a D3Q19 model. The set of model parameters used for the IGLB model is as follows:

$s_{5}=s_{11}=s_{17}=1.2$,
$s_{2}=s_{5}-0.1=1.1$,
$s_{3}=s_{2}-0.1=1.0$.

The relaxation parameters $s_{9}=s_{13}$ are determined by the viscosity from Eq.

(22).

Although the computations are performed with a fully explicit scheme in time, only steady state solutions are presented here. In order to define steady state situation, the following expression is used:

$\delta=\sqrt{\frac{1}{N} \sum_{i=1}^{N}\left(u^{n+1}-u^{n}\right)^{2}+\left(v^{n+1}-v^{n}\right)^{2}+\left(\mathrm{w}^{n+1}-\mathrm{w}^{n}\right)^{2}}$,

where $N$ is the total number of nodes in the solution domain; $n$ is old time level; and $\left(u^{n}, u^{n+1}\right),\left(v^{n}, v^{n+1}\right)$, and $\left(w^{n}, w^{n+1}\right)$ are $\mathrm{x}, \mathrm{y}$ and $\mathrm{z}$ components of the velocity for the old and new time levels, respectively. The steady-state results are obtained when $\delta$ becomes smaller than $10^{-10}$.

Figure 3 shows time history of relative error of the velocity distribution at Reynolds number of $R e=100,400$, and 1000. From Fig. 3, it can be observed that both IBGKLB and IGLB models need approximately the same time step or iteration number to reach the steady state at $R e=100$. Furthermore, for $R e=400$ and 1000, the number of iteration for IGLB model, which is required to achieve the steady sate, is more than that of IBGKB model.

The grid dependency studies for cases of $R e=100,400$, 1000 are conducted first. The mesh size in the computational domain is varied from $21 \times 21 \times 21$ to $81 \times 81 \times 81$. The results of the grid dependency study are exhibited in Table 3 in terms of $u_{\min }, w_{\min }, w_{\max }$ at the mid-plane of $Y=0.5$. In addition, Figs. 4 and 5 display the $\mathrm{x}$ and $\mathrm{z}$ components of the velocity distributions along the vertical and horizontal centerline of the cavity for $R e=400$ and 1000 at different mesh sizes, respectively. As shown in Table 3 and in Figs. 4 and 5, with increasing of mesh size from $21 \times 21 \times 21$ to $81 \times 81 \times 81$, the differences between presented IGLB results are decreased from $2.44 \%$ to $0.47 \%, 1.29 \%$ to $0.13 \%$, and $10.10 \%$ to $0.39 \%$ for Reynolds numbers of $R e=100,400$, and 1000, respectively. These findings manifest that the utilization of $81 \times 81 \times 81$ mesh points by IGLB model can produce very 
accurate numerical results, hence it is used in the numerical simulations.

Figures 6 to 11 present the velocity profiles of the $u$ component on the vertical central line and the $\mathrm{w}$ component on the horizontal central line on the plane of $Y=0.5$ in a cubic cavity for $R e=100,400$ and 1000, respectively. As shown in those figures, all the IGLB results are in good agreement with both IBGKLB and Ku's results (Ku et al. 1987)

Figures 12 to 17 illustrate the comparison of $\mathrm{x}$ and $\mathrm{z}$ components of the velocity distributions very close to the left vertical wall ( $i=2$ lattice point) for IBGKLB and IGLB models at various Reynolds numbers $(R e=100,400$ and 1000). Results clearly show that the velocity distributions (both $\mathrm{x}$ and $\mathrm{z}$ components) by IBGKLB model present obvious spatial oscillations close to the upper-left corner, while the velocity distributions by IGLB model display much less spatial oscillations in the same region of interest.

In general, the spatial oscillations of solution around the upper-left corner intensify as the Reynolds number increases. The difference represents that IGLB model is more suitable, as compared to IBGKLB model, for simulation of fluid flow around geometrical singularity and potentially higher Reynolds-number fluid flows.

Figures 18 to 20 show the pressure contours at middle plane of $Y=0.5$ for different models at Reynolds numbers of 100, 400 and 1000. The pressure contours near the top corners show that the IGLB results have less spatial oscillation, and have improved the quality of the flow field which is more significant at higher Reynolds numbers.

Figure 21 reveals the streamlines projected onto three orthogonal mid-planes of (a) $Z=0.5$, (b) $X=0.5$ and (c) $Y$ $=0.5$ for $R e=100,400$ and 1000 , respectively. Notice that the streamlines are created by the velocity vectors on the pertinent mid-plane. Therefore, they can be regarded as projection of streamlines. As it is seen in the plots of $Y=$ 0.5 for $R e=100,400$ and 1000 , the 3-D results are quite different from the 2-D ones due to the boundary layer effect by the lateral wall. Although the streamlines in the mid plane of $Y=0.5$ are similar to those of a twodimensional case, the strength of the main vortex is decreased. As Reynolds number is increased from 100 to 1000 , the main vortex gradually moves toward the center and its strength is also intensified. Additionally, a second vortex is formed at the lower right corner at $\mathrm{Re}=1000$.

For the plots of $Z=0.5$ and $X=0.5$, it is observed that, above $R e=400$, a couple of transversal vortices are produced near the lower right and left corners at each plane, and with an increase of the Reynolds number, their locations gradually move to the lower bottom wall. Furthermore, their strengths are enhanced.

By accurately monitoring plots of $X=0.5$ for $R e=100$, 400 , and 1000 , one can see that a pair of secondary vortices at the corners of the top wall becomes more obvious over
$R e=400$. Besides, for $R e=1000$, as shown in plot of $X=$ 0.5 , the vortices at the bottom wall are observed to bulge to the center, demonstrating the formation of a couple of Taylor-Görtler-like (TGL) vortices. The TGL vortices are formed due to the concave surface produced by the downstream secondary vortex at the bottom of the cavity (mid-plane of $Y=0.5$ ).

Table 4 presents the locations of the main vortex core at the mid-plane of $Y=0.5$ and the transversal vortex core at the mid-plane of $X=0.5$ between the proposed IGLB results and those of $\mathrm{Ku}$ et al. (1987). From this table, one can observe that our GLB results conform well to those of Ku et al. (1987).

The numerical experiments show that when the Reynolds number is increased to 2000 , the pressure field obtained by using the IGLB scheme still bears useful information, at least at some distance from the top corner singularities. In contrast with the entire pressure field acquired by IBGKLB model conveys the severe spurious oscillations at $R e=$ 2000. As the Reynolds number is increased up to 4000, in which the flow field becomes unsteady and complex threedimensional vortex shedding is observed, the IBGKLB code blows up due to the numerical instability while the IGLB code is stable although the results are not convergent to the required criteria.

Figures 22 and 23 display the results of IGLB model for time step of 225000 at Reynolds number of $R e=4000$. By noticing Fig. 23, the TGL vortices can be observed in midplane of $Z=0.5$ and $X=0.5$.

In order to study the stability of the method, we perform high Reynolds number simulations with a smaller grid size. It is found that the present method can perform simulations at low values of the viscosity (thus, high Reynolds number) without any numerical instability.

As the width of the system is approximately 50 lattice units, the Reynolds number is set by varying the viscosity. We compute the lower bounds of the viscosity for this particular flow by using the IGLB and IBGKLB schemes. The lower bounds are $1.0 \times 10^{-3}$ for the IGLB scheme and $2.5 \times 10^{-3}$ for the IBGKLB scheme with identical discretization, and initial and boundary conditions; Viscosities smaller than these bounds lead to numerical instability in the simulation. Hence for our test problem with the same grid size, the maximum Reynolds number attainable by using the IGLB model is about 2.5 times that achievable using the IBGKLB model.

The number of sites updated per second of the D3Q19IGLB method for our test case conducted using a personal computer with an AMD Athlon (tm) $643200+2.01 \mathrm{GHz}$ processor is about $0.149862 \times 10^{6}$ in comparison with $0.155774 \times 10^{6}$ for the D3Q19-IBGKLB method. Therefore, the IGLB scheme is approximately 4\% slower than the IBGKLB counterpart. Hence, the computational overhead due to the projections between $\mathbf{V}$ and $\mathbf{M}$ is not heavy. It is generally about $5 \%$ of the IBGKLB algorithm. However, it is important to mention that, with the same 
computational effort and near the limit of numerical stability, the results obtained by using the IGLB scheme are more accurate.

\subsubsection{Backward-Facing Step Flow}

The backward-facing step flow is a second well documented test case (Chiang et al. 1999). By imposing periodic conditions on the front and back faces of a threedimensional channel (c.f. Fig. 24), one may simulate the two-dimensional flow using a three-dimensional geometry and flow solver. To perform this simulation, the grid dependency study is performed and a suitable lattice $(945 \times 13 \times 63)$ is appointed. At the channel inlet, a quadrature velocity profile is imposed. At the outlet flow boundary, a fixed pressure is imposed and velocity components are extrapolated downstream. Furthermore, the no-slip boundary condition is imposed on the solid walls.

The size of the step is such that $S / H=31 / 63$ (c.f. Fig. 24), and the Reynolds number is defined by: $\operatorname{Re}=4 U(H-S) / 3 v$ (Chiang et al. 1999), which is 200 here. In this definition, $U$ is the maximum inlet velocity.

One of the most representative and sensitive flow characteristics is the recirculation zone length. Table 5 presents the results for the normalized recirculation zone length $(x / S)$. Considering the presented results in Table 5, it is shown that the result of IGLB model is more accurate than that of IBGKLB model. Also, the slight discrepancy between present results and those of Ref. (Chiang et al. 1999) can be due to the slight difference between $S / H$ values in the channel geometry. In the present model $S / H=31 / 63$, whereas in Ref. (Chiang et al. 1999) $S / H=49 / 101$.

The results for the backwards-facing step flow at $R e=200$ are shown in Figs. 25 to 29. Figures 25 and 27 show the pressure contours at $Y=0.5$ for the D3Q19 IBGKLB and IGLB models, respectively. Also, Figs. 26 and 28 show the pressure contours near the step. Once again, it is shown that the contour lines are much smoother near the corners for the IGLB model, which demonstrates the improved stability of the IGLB model.

\subsubsection{Double Shear Flow}

The double shear flow is a standard test case to check the accuracy of different schemes used in incompressible flows (Di et al. 2005). This problem is governed by the incompressible Navier-Stokes equations. The shear layers are perturbed at the initial time. The initial perturbation evolves with time and eventually large vertical structures appear. The initial conditions are given by:

$$
\begin{aligned}
& u_{0}(x, z)=\left\{\begin{array}{lc}
\tanh (\kappa(z-0.25)), & z \leq 0.5, \\
\tanh (\kappa(0.75-z)), & z>0.5,
\end{array}\right. \\
& w_{0}(x, z)=\delta \sin (2 \pi x),
\end{aligned}
$$

where $(x, z) \in\left(\begin{array}{ll}0 & 1\end{array}\right)$. The parameter $k$ and $\delta$ represent the shear layer width and the size of the perturbation, respectively. In our computations, the shear layer width is $k$ $=30$ and the perturbation size used is $\delta=0.05$. In order to the grid dependency study, three meshes such as $416 \times 6 \times 416,576 \times 6 \times 576$, and $768 \times 6 \times 768$ are used. As shown in Figs. 30 to 33, both $\mathrm{x}$ and $\mathrm{z}$ components of the velocity vectors obtained by $576 \times 6 \times 576$ and $768 \times 6 \times 768$ have good conformity with each other. So, a $576 \times 6 \times 576$ lattice is selected in the simulation. The Reynolds number defined as that of the previous test case is 10,000 . Furthermore, the periodic boundary condition is used for all variables on the boundaries in the all directions, i.e., $x$, $\mathrm{y}$, and $\mathrm{z}$ directions.

Figures 34 and 35 show the vorticity contours, $\omega=\left(\partial_{x} u_{z}-\partial_{z} u_{x}\right)$, the pressure contours, and the streamlines of the double shear flow at $t=0.8$, where layer width parameter, $k$, is 30 , viscosity, $v$, is $1 / 10,000$, respectively. The same contour levels are used to create all the figures. As the vorticity contours are illustrated in Fig. 34, both proposed generalized lattice Boltzmann and the moving mesh finite element methods (Di et al. 2005) produce the same structure.

\subsection{Simulation of Thermally Driven Flows}

\subsubsection{Simulation of Natural Convection in a Side-Heated Cubic Cavity}

Numerical simulation for the natural convection flow in a side-heated cubic cavity is carried out using the extension of the proposed IGLB method for simulation of thermal flow problems, i.e. Hybrid Thermal IGLB (HTIGLB) model. Figure 36 shows a schematic configuration of the setup in the simulation. No-slip boundary conditions are imposed on all the faces of the cube. Two opposite vertical walls of cube are maintained respectively at $+T_{0}$ (for $x=$ 0.5 ) and $-T_{0}$ (for $x=L_{x}-0.5$ ). The four other faces are adiabatic. With respect to the LB scheme, we use the bounce back scheme at all solid walls to satisfy the no-slip condition together with the extrapolation scheme to obtain values for $T$ at the boundaries. Initially, the fluid is at rest and a temperature field is varied linearly from the hot plate to the cold plate.

The temperature difference between the hot and cold walls introduces a temperature gradient in a fluid, and the consequent density difference induces a fluid motion, that is, convection. In the simulation, the Boussinesq approximation is applied to the buoyancy force term.

$r \mathbf{G}=\operatorname{rbg}\left(T-T_{m}\right) \mathbf{j}$

where $\beta$ is the thermal expansion coefficient, $g$ is the acceleration due to gravity, $T_{m}$ is the average temperature and $\mathbf{j}$ is the vertical direction opposite to that of gravity. 
A. R. Rahmati and M. Ashrafizaadeh / JAFM, Vol. 2, No. 1, pp. 71-96, 2009.

The dynamical similarity depends on two dimensionless parameters: the Prandtl number $P r$ and the Rayleigh number $R a$ defined as:

$$
\operatorname{Pr}=\frac{u}{a}, R a=\frac{g b \mathrm{D} T L_{x}^{3}}{u a},
$$

where $L_{x}$ is the horizontal length of the cavity, and DT is the temperature difference between the hot and cold walls.

We carefully choose the characteristic velocity $V_{c}=\sqrt{g b \mathrm{D} T L_{x}} \quad$ so that the low-Mach-number approximation maintains.

Nusselt number $N u$ is one of the most important dimensionless parameters in describing the convective heat transport. The volume-averaged Nusselt number can be defined as the following relation:

$$
\begin{aligned}
N u= & \frac{L_{x}}{a \mathrm{D} T} \frac{1}{L_{x} L_{y} L_{z}} \\
& \grave{\mathrm{O}}_{1}^{L_{x}} \grave{\mathrm{O}}_{1}^{L_{y}} \grave{\mathrm{O}}_{1}^{L_{z}} q_{x}(x, y, z) d x d y d z,
\end{aligned}
$$

where $q_{x}(x, y, z)=u T(x, y, z)-a(\mathbb{\Phi} / \mathbb{\Phi} x) T(x, y, z)$ is the local heat flux in the horizontal direction.

A uniform grid is used throughout the current numerical simulations. The convergence criteria in each case are as follows:

$$
\sqrt{\frac{1}{N} \sum\left(u^{n+1}-u^{n}\right)^{2}+\left(v^{n+1}-v^{n}\right)^{2}+\left(\mathrm{w}^{n+1}-\mathrm{w}^{n}\right)^{2}} \leq 10^{-5},
$$

$$
\sqrt{\frac{1}{N} \sum\left(T^{n+1}-T^{n}\right)^{2}} \leq 10^{-5}
$$

In all simulations, $P r$ is set to be 0.71 , and due to the restriction of computer capability, the grid size of $127 \times 127 \times 127$ is used for $R a=10^{3}, 10^{4}, 10^{5}$, and $10^{6}$. In order to study the dependency of the calculations on the grid size, the numerical simulations for $R a=10^{4}$ using HTIGLB method are conducted on seven grid sizes: $21 \times 21 \times 21,41 \times 41 \times 41,51 \times 51 \times 51,61 \times 61 \times 61,81 \times 81 \times 81$, $101 \times 101 \times 101$, and $127 \times 127 \times 127$. The result is given in Table 6. This table contains the numerical result of the maximum of $\mathrm{x}$ and $\mathrm{z}$ components of the velocity in the cavity, with its location and the averaged Nusselt number at the entire system. It should be noted that the velocity shown in the table is normalized by the reference velocity of $\alpha / L_{x}$ (velocity reference of $V_{r e f}$ is given as $\left.a / L_{x}=\sqrt{g b \mathrm{D} T L_{x} / R a \operatorname{Pr}}\right)$. From the results presented in Table 6, it can be clearly observed that, as we increase the size of the grid, the difference between the computed quantities is alleviated from $6.253 \%$ to $0.625 \%$ for $u_{\max } / V_{\text {ref }}, 7.868 \%$ to $0.748 \%$ for $w_{\max } / V_{\text {ref }}$, and from $14.570 \%$ to $1.49 \%$ for Nusselt number. This indicates that the utilization of $127 \times 127 \times 127$ grid in our proposed model can give very accurate numerical results.

In Fig. 37, we show the time history of the Nusselt number for $R a=10^{4}$ and $10^{5}$. This figure shows that the required time step to reach the steady state for Rayleigh number of $R a=10^{5}$ is more than that of $R a=10^{4}$.

Figure 38 displays temperature contours and streamlines for the cubic differentially heated buoyant cavity flow in final steady states at middle plane of $Y=0.5$ for Rayleigh numbers of $R a=10^{3}, 10^{4}, 10^{5}$, and $10^{6}$, respectively. At $R a$ $=10^{3}$, streamlines are those of a single vortex. The center of which is in the center of the cavity. The corresponding temperature contours are parallel to both hot and cold walls, manifesting that most of the heat transfer mechanism is by heat conduction. As the Rayleigh number is enhanced to $R a=10^{4}$, the central streamline is deformed into an elliptic shape and the effects of convection can be observed in the temperature contours. At $R a=10^{5}$, the central streamline is lengthened and two secondary vortices emerge inside it. The temperature distributions become horizontal in the middle of the system, indicating that the dominant of heat transfer mechanism is convection. At $R a=10^{6}$, two secondary vortices get stronger and the temperature distributions become more horizontal at the center of the cavity. Consequently, with regard to these figures, as the Rayleigh number increases, the fluid motion takes place near the vertical walls and the flow in the core of the cavity becomes quasi-stationary. Additionally, when the Rayleigh number increases, two tendencies are perceived for the temperature contours; 1) an increase in the temperature gradients close to the hot and cold boundaries, and 2) intensified mixing of the hot and cold fluids. Both inclinations enhance the heat transfer in the flow domain.

Table 7 presents the simulated results obtained for the Nusselt number at Rayleigh numbers of $R a=10^{3}, 10^{4}, 10^{5}$, and $10^{6}$, respectively, using $128 \times 128$ grid system. The equivalent Nusselt data presented by Tric et al. (2000) are also shown in Table 7 for comparison purposes. From this table, it can be observed that our results agree well with those by Tric et al. (2000) for Rayleigh numbers less than $10^{4}$. At higher Rayleigh numbers, the current LB simulation slightly underestimates the heat transfer due to insufficient spatial resolution.

The above results indicate that our new 3-D hybrid thermal IGLB model is capable of solving the thermal flow problems.

\subsubsection{Simulation of Natural Convection from a Discrete Heat Source on the Bottom of a Horizontal Enclosure}

Natural convection (Bejan 1984) has been regarded as an efficient procedure for cooling electronic devices due to its high credibility, low maintenance cost, and absence of noise. Natural convection from vertical enclosures with discrete heat sources attached on the vertical wall (Mathews et al. 2007; Keyhani et al. 1988; Heindel et al. 1996; Linhui et al. 2006; Mobedi and Sunden 2006) has been given a sizeable attention. The problem of discrete 
heat source related to a horizontal surface of an electronic package (Deng et al. 2002; Sezai and Mohamad 2000; Ichimiya and Saiki 2005) is of at least comparable significance and found in various engineering applications, such as cooling of electronic equipment and airconditioning of buildings with chilled ceilings. From the point of view of the flow pattern and heat transfer features, natural convection of discrete heat source in horizontal enclosures can be much more complex than that happening in vertical enclosures or along vertical plates.

Polentini et al. (1993) explain that a horizontal arrangement of discrete heat sources is preferred when uniform heat transfer rates are wanted from diverse rows, which is most often the case for cooling of electronic components.

The natural convection from a discrete flush-mounted rectangular heat source on the bottom of a horizontal enclosure is similar to Raleigh-Bénard problem (Pallares et al. 1995, Ouertatani et al. 2008, Calcagni et al. 2005), which consists of an enclosure heated from below and cooled from top. The induced flow pattern includes rotating rolls or mushroom type construction depending on Rayleigh number, Prantdl number, and geometrical restrictions.

In order to study Rayleigh-Bénard convection, diverse numerical simulations have been conducted using different thermal LB models (Chen and Doolen 1998; Shan 1997; $\mathrm{Xu}$ and Lui 1999; Inamuro et al. 2002; Kao and Yang 2007). However, the heat transfer and natural convection due to a discrete heat source which is mounted flush with the bottom wall of a horizontal enclosure has not been inspected and analyzed by thermal LB models. The present study utilizes the proposed HTIGLB model to simulate the natural convection from a discrete heat source on the bottom wall of a horizontal enclosure.

Figure 39 displays the schematic illustration of the physical configuration and the coordinate system. The problem comprises a chip of constant surface temperature in an enclosure with the width of $L_{x}$, depth of $L_{y}$, and height of $H$. The aspect ratios $A R_{x}=L_{x} / H$ and $A R_{y}=L_{y} / H$ of the rectangular cavity are equal to 4 . The chip with surface temperature $T_{h o t}$ is on the bottom horizontal wall and has dimensions $l_{x}$ and $l_{y}$ in $\mathrm{x}$ and $\mathrm{y}$ directions respectively. The longitudinal aspect ratio $a r_{x}=l_{x} / H$ and the lateral aspect ratio $a r_{y}=l_{y} / H$ of the chip are unity. The bottom wall surface is presumed to be adiabatic except for the chip, while the upper wall surface is kept at temperature $T_{\text {cold }}$. The sidewalls of the enclosure are supposed to be adiabatic. The cooling fluid is air which has a Prandtl number of 0.71 . The Rayleigh numbers of $10^{3}$ and $10^{5}$ are considered in this research.

In LBM calculation, the characteristic velocity is assumed to be equal to 0.1 . Besides, to perform this problem, a $161 \times 161 \times 40$ lattice is used.

Figures 40 and 41 reveal the streamlines and the temperature contours of discrete heat source, which is set up flush with the bottom wall of the horizontal cavity, on the symmetry plane of $Y=0.5$ for Rayleigh numbers of $10^{3}$ and $10^{5}$. Furthermore, the results of Sezai and Mohamad (2000) are shown in these figures for comparison. As it is shown, the obtained results agree well with those of Sezai and Mohamad (2000). Note that the streamlines of mid plane of $Y=0.5$ are obtained from the $\mathrm{x}$ and $\mathrm{z}$ components of the velocity vector at that plane.

From Figs. 40 and 41, it can be observed that the flow pattern is identified by a single roll cell of nearly toroidal shape. As Rayleigh number increases from $10^{3}$ to $10^{5}$, centers of rotation in the two sections of the toroid moves towards lateral walls. This flow structure has a vertical symmetry axis which passes through the center of enclosure.

The hot fluid ascends in the central section due to buoyancy forces, until it reaches close to the top wall where it turns radially outward, towards the lateral walls while it is cooled. Then, it turns downward near the sidewalls. Ultimately, the limitation applied by the bottom wall enforces the fluid to turn radially inward, receiving heat when it approaches the discrete heat source. The flow path is completed as the colder fluid is entrained to the ascending flow at the center of the enclosure.

At Rayleigh number of $10^{3}$, heat transfer from the discrete heat source is dissipated by means of conductiondominated mechanism. For Rayleigh number of $10^{5}$, the buoyancy driven flow in the central area between the rolls deforms the temperature contours. Furthermore, the heat transfer becomes increasingly advection dominated.

Table 8 compares the positions of roll cell centers in the two sections of the toroid at the mid-plane of $Y=0.5$, between the proposed HTIGLB results and those of Sezai and Mohamad (2000). As shown in Table 8, our HTIGLB results agree well when compared to those of Sezai and Mohamad (2000) and the relative errors percentage between the two sets of results which are given in parentheses are less than $1.64 \%$ at $R a=10^{3}$ and $3.7 \%$ for $R a=10^{5}$.

Consequently, computations of natural convection from a discrete heat source on the bottom of a horizontal enclosure accurately predicted the flow characteristics for different Rayleigh numbers. The results also agree well with those of Sezai and Mohamad (2000). This shows that our 3-D HTIGLB model has the capability to simulate the thermally driven flow problems.

\section{CONCLUSION}

In this work, the compressibility effect of lattice Boltzmann methods on the simulation of incompressible flows has been investigated. Then, an incompressible generalized lattice Boltzmann method has been proposed for the simulation of three-dimensional flows.

The proposed IGLB model is validated by its application to simulate three-dimensional lid-driven flow in a cubic 
A. R. Rahmati and M. Ashrafizaadeh / JAFM, Vol. 2, No. 1, pp. 71-96, 2009.

cavity, a backward-facing step flow, and a double shear flow at different Reynolds numbers.

It is found that the obtained results agree very well with those from Navier-Stokes solvers. This demonstrates that our proposed IGLB model can simulate three-dimensional incompressible flows.

The stability of the model is also analyzed and compared with the IBGKLB model. It is found that the mechanism of separate relaxations for the kinetic modes results in a model which is much more stable than the BGK LB model. The proposed IGLB model compared to the IBGKLB model needs the transformations between the velocity space $\mathbf{V}$ and the moment space $\mathbf{M}$, back and forth in each step in the evolution equation. However, the extra computational cost due to this transformation is about $5 \%$ of the total computing time. Therefore the computational efficiency is similar to the IBGKLB model.

The proposed three-dimensional generalized lattice Boltzmann method is successfully extended and applied to simulation of the three-dimensional incompressible buoyancy-induced flows. The numerical results of the three-dimensional steady-state natural convection of air in a cubical enclosure obtained by HTIGLB method agree well with the existing benchmark data. Additionally, natural convection from a discrete heat source flushmounted on the bottom of a horizontal cavity is simulated. It is shows that the proposed scheme is very suitable for simulation of buoyant flow problems.

As mentioned above, the obtained results show that IGLB model is more accurate and stability compared to the incompressible BGK lattice Boltzmann method. Therefore, the current proposed method is potentially capable of simulating high Reynolds number flows which will be discussed in the subsequent work and a relevant turbulent model will be applied to the proposed method.

The principal advantage of lattice Boltzmann method, which has not been evaluated in this article and will be studied in the future work, is the high parallelism of such an algorithm. This provides a chance to use such a method for the simulation of higher complexity fluid flows. It makes lattice Boltzmann methods competitive tools in contrast to conventional CFD techniques.

It is important to note that the numerical tests considered here are simple, and our aim is just to validate the scheme. More demanding complex tests will be carried out in future work.

\section{REFERENCES}

Bejan, A. (1984). Convection Heat Transfer, Wiley, New York.

Bhatnagar, P.L., E.P. Gross, and M. Krook (1954). A model for collisional processes in gases I: small amplitude processes in charged and neutral onecomponent system. Physical Review 94, 511-524.
Boon, J.P., D. Dab, R. Kapral, and A. Lawniczak (1996). Lattice gas automata for reactive systems. Physics Reports 273, 55-148.

Bouzidi, M., D. d'Humières, P. Lallemand, and L.S. Luo (2001). Lattice Boltzmann equation on a twodimensional rectangular grid. Journal of Computational Physics 172, 704-717.

Calcagni, B., F. Marsili, and M. Paroncini (2005). Natural convective heat transfer in square enclosures heated from below. Applied Thermal Engineering 25, 25222531

Chen, H., S. Chen, and H.W. Matthaeus (1992). Recovery of the Navier-Stokes equations using a lattice-gas Boltzmann method. Physical Review A 45, 5339-5342.

Chen, S., S.P. Dawson, G.D. Doolen, D.R. Janecky, and A. Lawniczak (1995). Lattice methods and their applications to reacting systems. Computers and Chemical Engineering 19(6), 617-646.

Chen, S. and G.D. Doolen (1998). Lattice Boltzmann method for fluid flows. Annual Review of Fluid Mechanics 30, 329-364.

Chen, S. and G.D. Doolen (1998). A Novel Thermal Model for the Lattice Boltzmann Method in Incompressible Limit. Journal of Computational Physics 146, 282-300.

Chiang T.P., T.W.H. Sheu, and C.C. Fang (1999). Numerical investigation of vortical evolution in a backward-facing step expansion flow. Applied Mathematical Modelling 23, 915-932.

Deng, Q.H., G.F. Tang, Y. Li, and M.Y. Ha (2002). Interaction between discrete heat sources in horizontal natural convection enclosures. International Journal of Heat and Mass Transfer 45, 5117-5132.

D'Humières, D. (1992). Generalized lattice Boltzmann equations. In: B.D. Shizgal and D.P. Weaver (Eds.), Rarefied gas dynamics: theory and simulations, Progress in Astronautics and Aeronauttics 159, 450458.

D’Humières, D., I. Ginzburg, M. Krafczyk, P. Lallemand, and L.S. Luo (2002). Multiple-relaxation-time lattice Boltzmann models in three dimensions. Philosophical Transactions of the Royal Society of London A 360, 437-451.

Di Y., R. Li, T. Tang, and P. Zhang (2005). Moving mesh finite element methods for the incompressible NavierStokes equations. SIAM Journal on Scientific Computing 26(3), 1036-1056.

Du, R., B. Shi, and X. Chen (2006). Multi-relaxation-time lattice Boltzmann model for incompressible flow. Physics Letters A 359, 564-572. 
A. R. Rahmati and M. Ashrafizaadeh / JAFM, Vol. 2, No. 1, pp. 71-96, 2009.

Frisch, U., B. Hasslacher, and Y. Pomeau (1986). Hydrodynamics on Lattice Gases. Physical Review Letters 56, 1505-1508.

Guo, Z., B. Shi, and N. Wang (2000). Lattice BGK Model for Incompressible Navier-Stokes Equation. Journal of Computational Physics 165, 288-306.

He, X. and L.S. Luo (1997). Lattice Boltzmann Model for the Incompressible Navier-Stokes Equation. Journal of Statistical Physics 88(3), 927-944.

Heindel, T.J., F.P. Incropera, and S. Ramadyani (1996). Enhancement of natural convection heat transfer from an array of discrete heat sources. Intternational Journal of Heat Mass Transfer 39, 479-490.

Higuera, F.J. and J. Jimenez (1989). Boltzmann approach to lattice gas simulations. Europhysics Letters 9, 663668.

Hou, S. (1995). Lattice Boltzmann Method for incompressible Viscous Flow, $\mathrm{PhD}$ thesis at Kansas State University, Manhattan, Kansas.

Ichimiya, K. and H. Saiki (2005). Behavior of thermal plumes from two-heat sources in an enclosure. International Journal of Heat and Mass Transfer 48, 3461-3468.

Inamuro, T., M. Yoshino, H. Inoue, R. Mizuno, and F. Ogino (2002). A lattice Boltzmann method for a binary miscible fluid mixture and its application to a heattransfer problem. Journal of Computational Physics $179,201-215$.

Kao, P.H. and R.J. Yang (2007). Simulating oscillatory flows in Rayleigh-Bénard convection using the lattice Boltzmann method. International Journal of Heat and Mass Transfer 50, 3315-3328

Keyhani, M., V. Prasad, and R. Cox (1988). An experimental study of natural convection in a vertical cavity with discrete heat source. Journal of Heat Transfer 110(3), 616-624.

Ku H. C., R.S. Hirsh and T.D. Taylor (1987). A pseudospectral method for solution of the three-dimensional incompressible Navier-Stokes equations. Journal of Computational Physics 70(2), 439-462

Ladd, A.J.C. (1994). Numerical simulations of particulate suspensions via a discretized Boltzmann equation: Part 1. Theoretical foundation. Journal of Fluid Mechanics 271, 285-309.

Lallemand, P. and L.S. Luo (2000). Theory of the lattice Boltzmann method: dispersion, dissipation, isotropy, Galilean invariance, and stability. Physical Review E 61(6), 6546-6562.

Lallemand, P. and L.S. Luo (2003). Theory of the lattice Boltzmann method: acoustic and thermal properties in two and three dimensions. Physical Review E 68(3), 036706(1)-036706(25).

Lallemand P. And L.S. Luo (2003). Hybrid finitedifference thermal lattice Boltzmann equation. International Journal of Modern Physics B 17(1\&2), 41-47.

Linhui, C., T. Huaizhang, L. Yanzhong, Z. Dongbin (2006). Experimental study on natural convective heat transfer from a vertical plate with discrete heat sources mounted on the back. Energy Conversion and Management 47, 3447-3455

Mathews, R.N., C. Balaji, and T. Sundararajan (2007). Computation of conjugate heat transfer in the turbulent mixed convection regime in a vertical channel with multiple heat sources. Journal of Heat and Mass Transfer 43(10), 1063-1074.

McNamara, G.R. and G. Zanetti (1988). Use of the lattice Boltzmann to simulate lattice-gas automata. Physical Review Letters 61, 2332-2335.

Mezrhab A., M. Bouzidi, and P. Lallemand (2004). Hybrid lattice-Boltzmann finite-difference simulation of convective flows. Computers \& Fluids 33, 623-641.

Mobedi, M. and B. Sunden (2006). Natural convection heat transfer from a thermal heat source located in a vertical plate fin. International Communications in Heat and Mass Transfer 33, 943-950

Ouertatani N., N. Ben-Cheikh, B. Ben-Beya, and T. Lili (2008). Numerical simulation of two-dimensional Rayleigh-Bénard convection in an enclosure. Comptes Rendus Mecanique 336, 464-470

Pallares, J., I. Cuesta, F.X. Gran, and F. Giralt (1995). Natural convection in a cubical cavity heated from below at low Rayleigh numbers. International Journal of Heat Mass Transfer 39, 3233-3247.

Pan, C., M. Hilpert, and C.T. Miller (2004). LatticeBoltzmann simulation of two-phase flow in porous media. Water Resources Research 40, W01501, doi:10.1029/2003WR002120.

Peng, Y., C. Shu, and Y.T. Chew (2003). A 3D incompressible thermal lattice Boltzmann model and its application to simulate natural convection in a cubic cavity. Journal of Computational Physics 193, 260-274.

Polentini, M.S., S. Ramadhyani, and F.P. Incropera (1993). Single phase thermosyphon cooling of an array of discrete heat sources in a rectangular cavity. International Journal of Heat Mass Transfer 36, 39833996.

Qian, Y., D. d'Humières, and P. Lallemand (1992). Lattice BGK models for Navier-Stokes equation. Europhysics Letters 17, 479-484. 
A. R. Rahmati and M. Ashrafizaadeh / JAFM, Vol. 2, No. 1, pp. 71-96, 2009.

Rahmati, A.R., and M. Ashrafizaadeh (2007, May). Performance evaluation of multi relaxation time lattice boltzmann method for 3D fluid flow simulation. Proceedings of the $15^{\text {th }}$ annual international conference on mechanical engineering, Tehran, Iran, Amirkabir University of Technology Press, 173.

Rahmati A.R., M. Ashrafizaadeh, and E. Shirani (2008, February). Numerical simulation of viscous flows using an incompressible lattice Boltzmann method on non-uniform grids. Proceedings of the $7^{\text {th }}$ Iranian Aerospace Society Conference, Tehran, Iran, Sharif University of Technology Press, 43.

Rahmati, A.R., M. Ashrafizaadeh, and E. Shirani (2008, May). Natural Convection Simulation by Using Two Thermal Lattice Boltzmann Models. Proceedings of the $16^{\text {th }}$ annual international conference on mechanical engineering, Kerman, Iran, Shahid Bahonar University of Kerman Press, 109.

Rahmati, A.R., M. Ashrafizaadeh, and E. Shirani (2008, May). Improvement of Numerical Instability of Lattice Boltzmann Methods Using Various Techniques. Proceedings of the $16^{\text {th }}$ annual international conference on mechanical engineering, Kerman, Iran, Shahid Bahonar University of Kerman Press, 115.

Rahmati, A.R., M. Ashrafizaadeh, and E. Shirani (2008, May). Multi-Relaxation-Time Lattice Boltzmann Method for LES of Turbulent Flows. Proceedings of the $11^{\text {th }}$ Fluid Dynamics Conference, Tehran, Iran, K. N. Toosi University of Technology Press, 8.

Rahmati, A.R., M. Ashrafizaadeh, and E. Shirani (2008, August). Numerical Instability Analysis of the Lattice Boltzmann Equation Methods Using Different Schemes. Proceedings of the $12^{\text {th }}$ Asian Congress of Fluid Mechanics, Daejeon, Korea, 33.

Rahmati, A.R., M. Ashrafizaadeh, and E. Shirani (2008, August). Incompressible Multi-Relaxation-Time LBM with Non-Uniform mesh for LES of Rayleigh-Bénard Convection Flow. Proceedings of the $12^{\text {th }}$ Asian Congress of Fluid Mechanics, Daejeon, Korea, 54.

Rahmati, A.R., M. Ashrafizaadeh, and E. Shirani (2008, June). Convective flow simulation by using two hybrid finite-difference thermal lattice Boltzmann models.
Book of Extended Abstract of the $5^{\text {th }}$ International Conference for Mesoscopic Methods in Engineering and Science (ICMMES), Amsterdam, The Netherlands.

Sezai, I. and A.A. Mohamad (2000). Natural convection from a discrete heat source on the bottom of a horizontal enclosure. International Journal of Heat and Mass Transfer 43, 2257-2266.

Shan, X. (1997). Simulation of Rayleigh-Bénard convection using a lattice Boltzmann method. Physical Review E, 55(3), 2780-2788.

Shan, X. and H. Chen (1993). Lattice Boltzmann model for simulating flows with multiple phases and components. Physical Review E 47(3), 1815-1819.

Teixeira C, H. Chen, and D.M. Freed (2000). Multi-speed thermal lattice Boltzmann method stabilization via equilibrium under-relaxation. Computer Physics Communications 129(1), 207-226.

Tric, E., G. Labrosse, and M. Betrouni (2000). A first incursion into the 3D structure of natural convection of air in a differentially heated cubic cavity, from accurate numerical solutions. International Journal of Heat and Mass Transfer 43, 4043-4056.

van Treeck, C., E. Rank, and M. Krafczyk (2006). Extension of a hybrid thermal LBE scheme for largeeddy simulations of turbulent convective flows. Computers \& Fluids 35(8), 863-871.

Wolfram, S. (1986). Cellular automaton fluids 1: Basic theory. Journal of Statistical Physics 45, 471-526.

Xu, K., and S.H. Lui (1999). Rayleigh-Bénard simulation using the gas-kinetic Bhatnagar-Gross-Krook scheme in the incompressible limit. Physical Review E 60(1), 464-470.

Zheng, H.W., C. Shu, and Y.T. Chew (2006). A lattice Boltzmann model for multiphase flows with large density ratio. Journal of Computational Physics 218(1), 353-371. 
A. R. Rahmati and M. Ashrafizaadeh / JAFM, Vol. 2, No. 1, pp. 71-96, 2009.

Table 1 Minimum x-component velocity for $R e=400$.

\begin{tabular}{|c|c|c|c|}
\hline$U_{0}$ & $u_{\text {CBGKLB }}$ & $u_{\text {IBGKLB }}$ & $u_{\text {ku et al. }}$ \\
\hline 0.1 & -0.236514 & -0.236678 & -0.241597 \\
\hline 0.15 & -0.236189 & -0.236536 & -0.241597 \\
\hline
\end{tabular}

Table 2 Minimum and maximum z-component velocity for $R e=400$.

\begin{tabular}{|c|c|c|c|}
\hline$U_{0}$ & $w_{\text {CBGKLB }}$ & $w_{\text {IBGKLB }}$ & $w_{\text {ku et al. }}$ \\
\hline \multirow{2}{*}{0.1} & Max $: 0.2058725$ & Max $: 0.2060855$ & Max $: 0.2081399$ \\
& Min: -0.37169405 & Min: -0.37212809 & Min: -0.3790700 \\
\hline \multirow{2}{*}{0.15} & Max $: 0.20548716$ & Max $: 0.205942258$ & Max $: 0.2081399$ \\
& Min: -0.3711390 & Min $:-0.372070342$ & Min: -0.3790700 \\
\hline
\end{tabular}

Table 3 Grid dependency study of cubic cavity driven flow for $R e=100,400$ and 1000 .

\begin{tabular}{|c|c|c|c|c|c|}
\hline Reynolds no. & Mesh size & $21 \times 21 \times 21$ & $41 \times 41 \times 41$ & $61 \times 61 \times 61$ & $81 \times 81 \times 81$ \\
\hline \multirow{3}{*}{100} & $u_{\min }$ & -0.2049 & -0.2099 & -0.2119 & -0.2129 \\
\cline { 2 - 6 } & $w_{\min }$ & -0.2087 & -0.2294 & -0.2358 & -0.2392 \\
\cline { 2 - 6 } & $w_{\max }$ & 0.1301 & 0.1411 & 0.1449 & 0.1469 \\
\hline \multirow{3}{*}{400} & $u_{\min }$ & -0.2406 & -0.2375 & -0.2367 & -0.2364 \\
\cline { 2 - 6 } & $w_{\min }$ & -0.3306 & -0.3596 & -0.3679 & -0.3720 \\
\cline { 2 - 6 } & $w_{\max }$ & 0.1998 & 0.2039 & 0.2051 & 0.2058 \\
\hline \multirow{3}{*}{1000} & $u_{\min }$ & -0.3187 & -0.2866 & -0.2825 & -0.2814 \\
\cline { 2 - 6 } & $w_{\min }$ & -0.3970 & -0.4155 & -0.4223 & -0.4275 \\
\cline { 2 - 6 } & $w_{\max }$ & 0.2806 & 0.2520 & 0.2476 & 0.2466 \\
\hline
\end{tabular}

Table 4 Positions of the main vortex (mid-plane of $Y=0.5$ ) and the transversal vortex (mid-plane of $X=0.5$ ) centers.

\begin{tabular}{|c|c|c|c|c|c|c|c|}
\hline Reynolds no. & & \multicolumn{2}{|c|}{100} & \multicolumn{2}{c|}{400} & \multicolumn{2}{c|}{1000} \\
\hline & & Present work & Ku et al. & Present work & Ku et al. & Present work & Ku et al. \\
\hline $\begin{array}{c}\text { Main vortex } \\
\text { in mid-plane of } \\
Y=0.5 .\end{array}$ & $\mathrm{z}$ & 0.765 & 0.75 & 0.578 & 0.6 & 0.468 & 0.5 \\
\cline { 2 - 9 } & $\mathrm{x}$ & 0.624 & 0.6 & 0.626 & 0.633 & 0.597 & 0.61 \\
\hline $\begin{array}{c}\text { Transversal vortex } \\
\text { in mid-plane of } \\
X=0.5 .\end{array}$ & $\mathrm{z}$ & 0.355 & 0.33 & 0.202 & 0.204 & 0.133 & 0.136 \\
\cline { 2 - 9 } & $\mathrm{y}$ & 0.378 & 0.378 & 0.225 & 0.233 & 0.155 & 0.167 \\
\hline
\end{tabular}

Table 5 Dimensionless recirculation zone length for flow over backward-facing step.

\begin{tabular}{|c|c|c|c|c|}
\hline Model & IBGKLB model & IGLB model & Chiang's work & Reynolds number \\
\hline$x / S$ & 5.252 & 5.266 & 5.359 & 200 \\
\hline
\end{tabular}


A. R. Rahmati and M. Ashrafizaadeh / JAFM, Vol. 2, No. 1, pp. 71-96, 2009.

Table 6 Comparison of numerical results for $R a=10^{4}$ and different grid sizes.

\begin{tabular}{|c|c|c|c|c|c|c|c|}
\hline Grid size & $21 \times 21 \times 21$ & $41 \times 41 \times 41$ & $51 \times 51 \times 51$ & $61 \times 61 \times 61$ & $81 \times 81 \times 81$ & $101 \times 101 \times 101$ & $127 \times 127 \times 127$ \\
\hline$u_{\max } / V_{r e f}$ & 14.474 & 15.379 & 15.527 & 15.613 & 15.760 & 15.842 & 15.938 \\
\hline$x / l x$ & 0.5 & 0.5 & 0.5 & 0.5 & 0.5 & 0.5 & 0.5 \\
\hline$y / l y$ & 0.5 & 0.5 & 0.5 & 0.5 & 0.5 & 0.5 & 0.5 \\
\hline$z / l z$ & 0.85 & 0.829 & 0.823 & 0.820 & 0.827 & 0.822 & 0.827 \\
\hline$w_{\max } / V_{r e f}$ & 15.861 & 17.109 & 17.389 & 17.569 & 17.778 & 17.918 & 18.046 \\
\hline$x / l x$ & 0.143 & 0.146 & 0.137 & 0.131 & 0.123 & 0.129 & 0.126 \\
\hline$y / l y$ & 0.5 & 0.5 & 0.5 & 0.5 & 0.5 & 0.5 & 0.5 \\
\hline$z / l z$ & 0.5 & 0.5 & 0.5 & 0.5 & 0.5 & 0.5 & 0.5 \\
\hline$N u$ & 1.537 & 1.761 & 1.818 & 1.859 & 1.914 & 1.948 & 1.975 \\
\hline
\end{tabular}

Table 7 Volume-averaged Nusselt number computed for different $R a$.

\begin{tabular}{|c|c|c|c|}
\hline Rayleigh no. & Grid size & Present work & Tric's work \\
\hline 1000 & $127 \times 127 \times 127$ & 1.07 & 1.07 \\
\hline 10000 & $127 \times 127 \times 127$ & 1.975 & 2.0542 \\
\hline 100000 & $127 \times 127 \times 127$ & 3.940 & 4.337 \\
\hline 1000000 & $127 \times 127 \times 127$ & 7.291 & 8.641 \\
\hline
\end{tabular}

Table 8 Positions of roll cell centers in the two sections of the toroid at the mid-plane of $Y=0.5$.

\begin{tabular}{|c|c|c|c|c|c|}
\hline \multirow[t]{2}{*}{ Rayleigh no. } & & \multicolumn{2}{|r|}{1000} & \multicolumn{2}{|c|}{100000} \\
\hline & & Present work & $\begin{array}{l}\text { Sezai and Mohamad } \\
(2000)\end{array}$ & Present work & $\begin{array}{l}\text { Sezai and Mohamad } \\
(2000)\end{array}$ \\
\hline \multirow{2}{*}{$\begin{array}{c}\text { Left roll cell } \\
\text { center in mid- } \\
\text { plane of } Y=0.5 \text {. }\end{array}$} & $\mathrm{x}$ & $\begin{array}{c}1.180 \\
(0.77 \%) \\
\end{array}$ & 1.171 & $\begin{array}{c}0.573 \\
(3.7 \%) \\
\end{array}$ & 0.595 \\
\hline & $\mathrm{z}$ & $\begin{array}{c}0.495 \\
(1.64 \%) \\
\end{array}$ & 0.487 & $\begin{array}{c}0.562 \\
(0.54 \%) \\
\end{array}$ & 0.559 \\
\hline \multirow{2}{*}{$\begin{array}{l}\text { Right roll cell } \\
\text { center in mid- } \\
\text { plane of } Y=0.5 \text {. }\end{array}$} & $\mathrm{x}$ & $\begin{array}{c}2.869 \\
(1.63 \%)\end{array}$ & 2.823 & $\begin{array}{c}3.477 \\
(2.54 \%)\end{array}$ & 3.391 \\
\hline & z & $\begin{array}{c}0.495 \\
(1.64 \%) \\
\end{array}$ & 0.487 & $\begin{array}{c}0.562 \\
(0.54 \%) \\
\end{array}$ & 0.559 \\
\hline
\end{tabular}




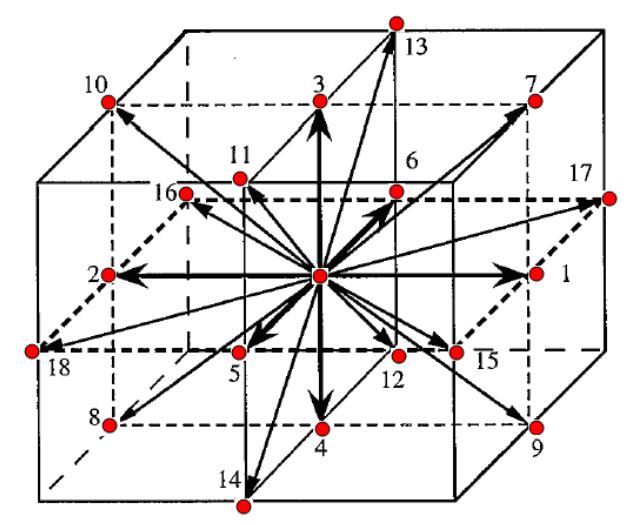

Fig. 1. D3Q19 lattice used in this work.

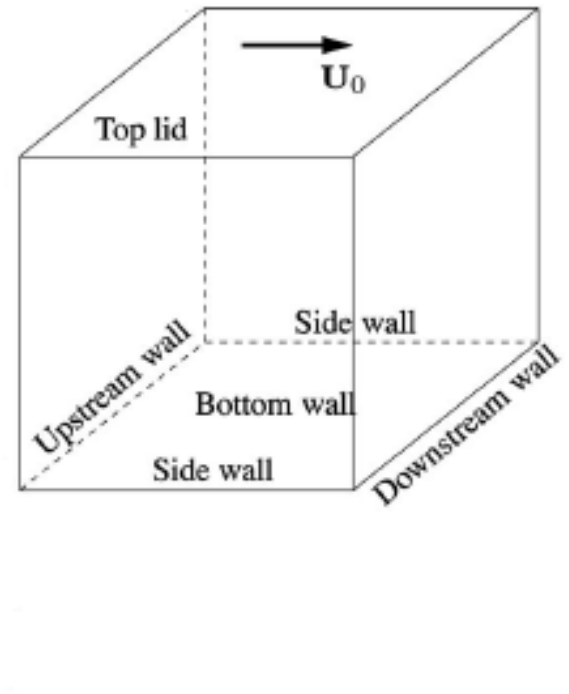

Fig. 2. Configuration of 3-D lid-driven cavity flow problem including the boundary conditions.

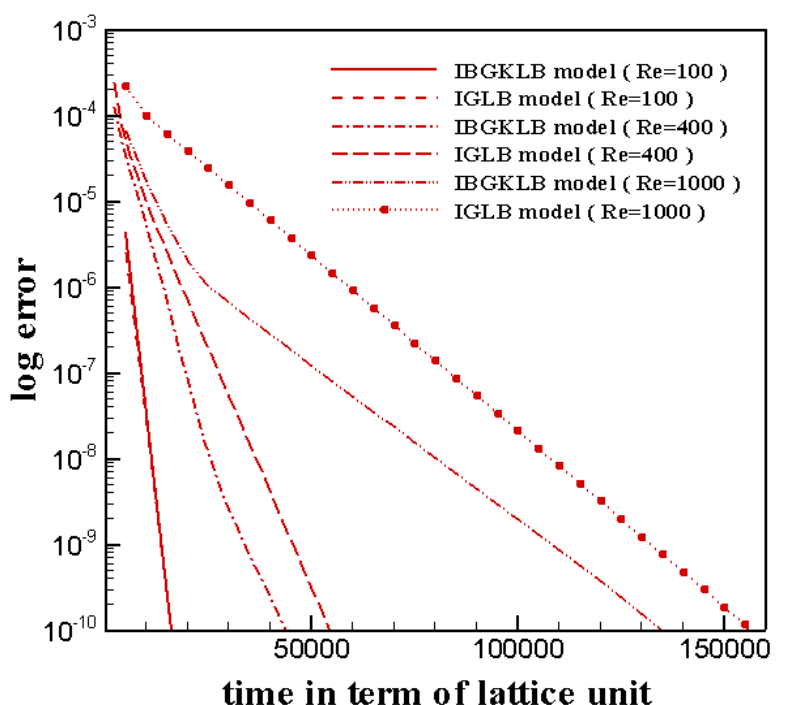

Fig. 3. Time history of the relative error of the velocity profile for different Reynolds numbers.

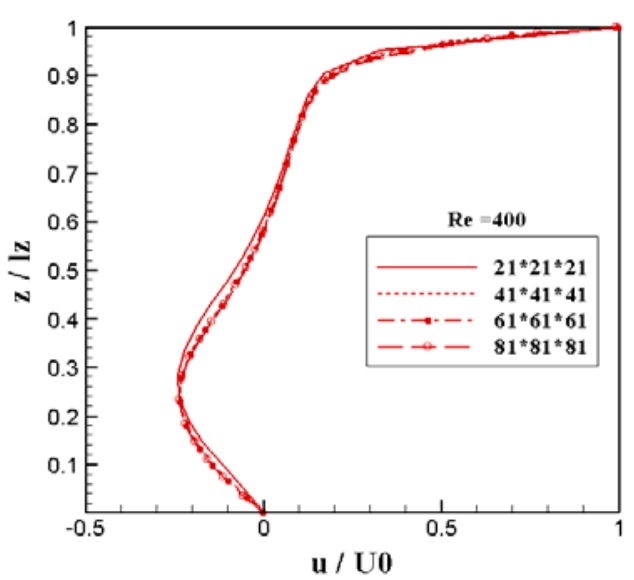

Fig. 4. X-component velocity along the cubic cavity vertical centerline for different mesh sizes.

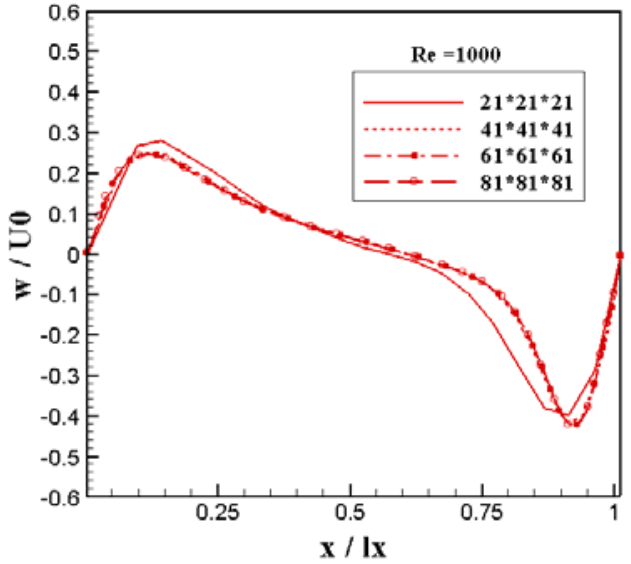

Fig. 5. Z-component velocity along the cubic cavity horizontal centerline for different mesh sizes. 
A. R. Rahmati and M. Ashrafizaadeh / JAFM, Vol. 2, No. 1, pp. 71-96, 2009.

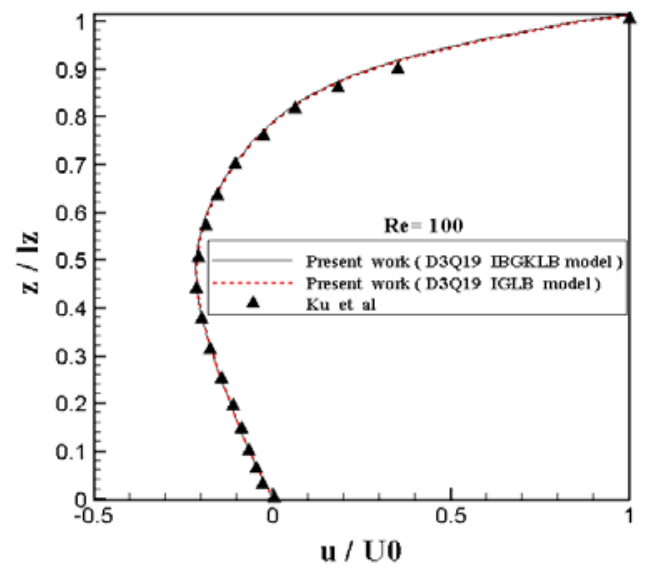

Fig. 6. X-component velocity along the cubic cavity vertical centerline for $R e=100$.

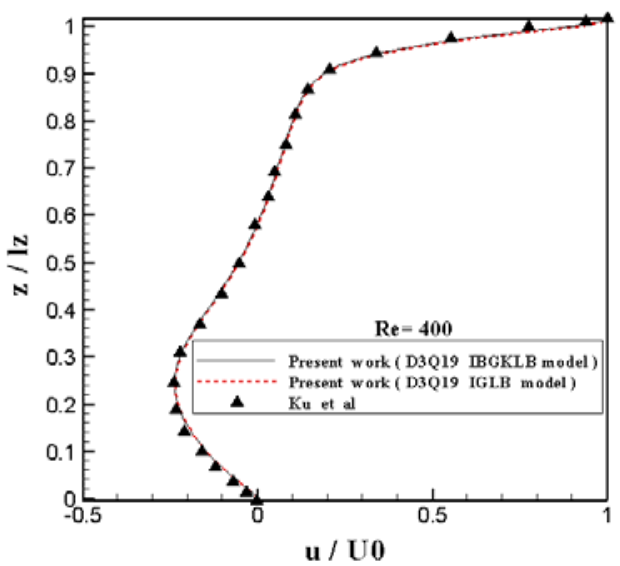

Fig. 8. X-component velocity along the cubic cavity vertical centerline for $R e=400$.

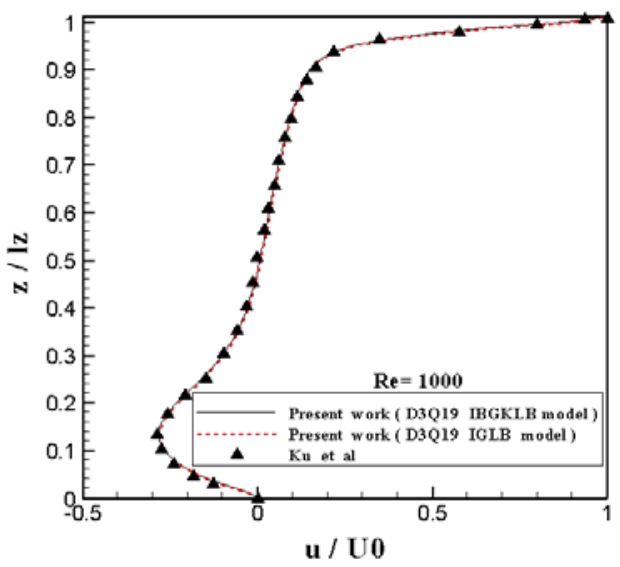

Fig. 10. X-component velocity along the cubic cavity vertical centerline for $R e=1000$.

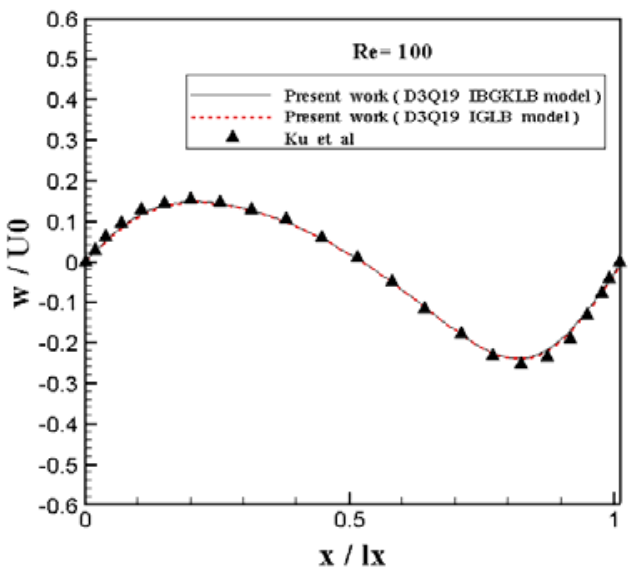

Fig. 7. Z-component velocity along the cubic cavity horizontal centerline for $R e=100$.

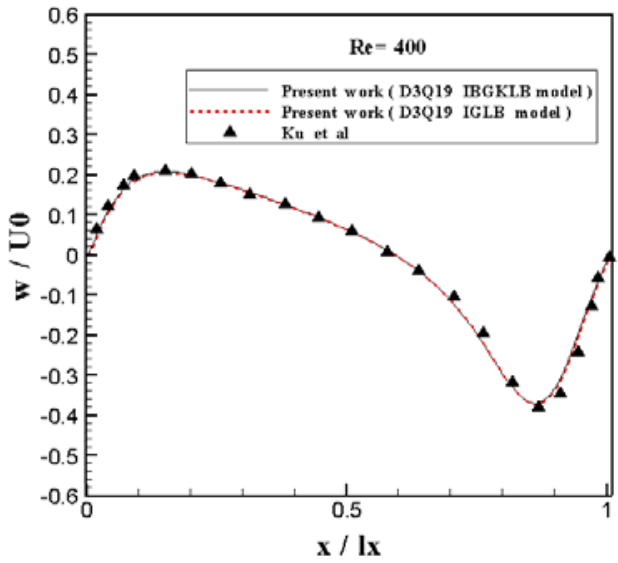

Fig. 9. Z-component velocity along the cubic cavity

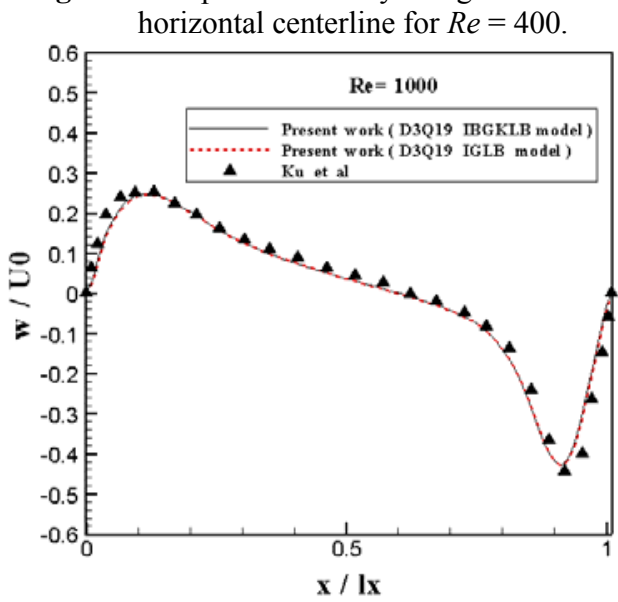

Fig. 11. Z-component velocity along the cubic cavity horizontal centerline for $R e=1000$. 
A. R. Rahmati and M. Ashrafizaadeh / JAFM, Vol. 2, No. 1, pp. 71-96, 2009.

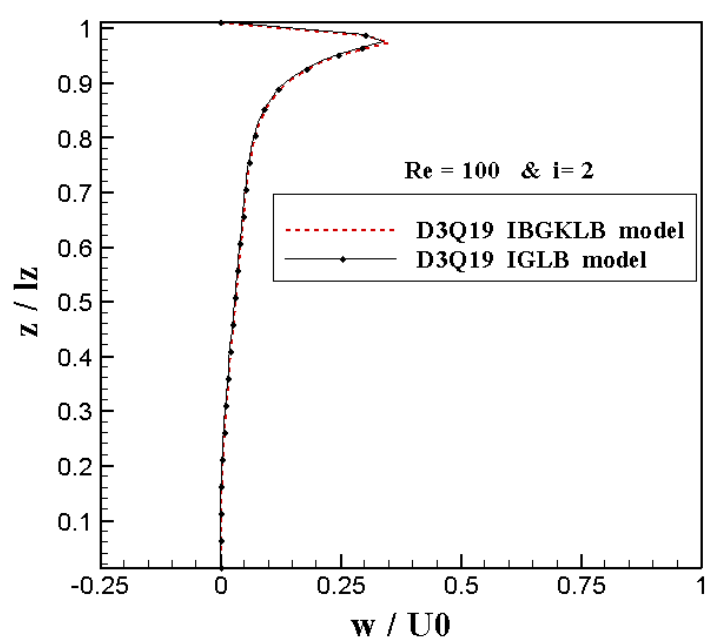

Fig. 12. X-component velocity profiles at $i=2, R e=$ 100.

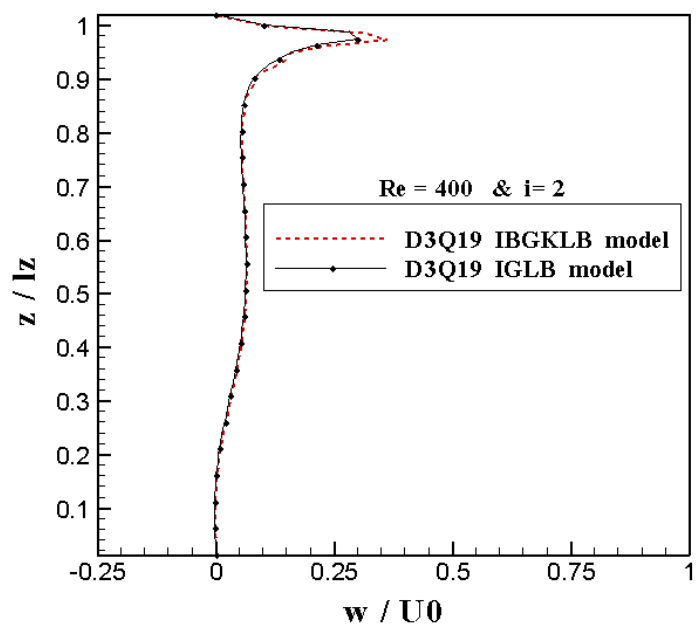

Fig. 14. X-component velocity profiles at $i=2, R e=$ 400 .

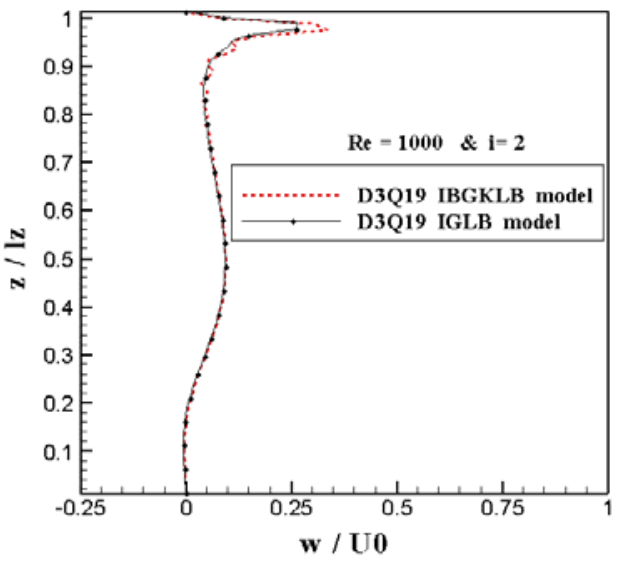

Fig. 16. X-component velocity profiles at $i=2, R e=$ 1000 .

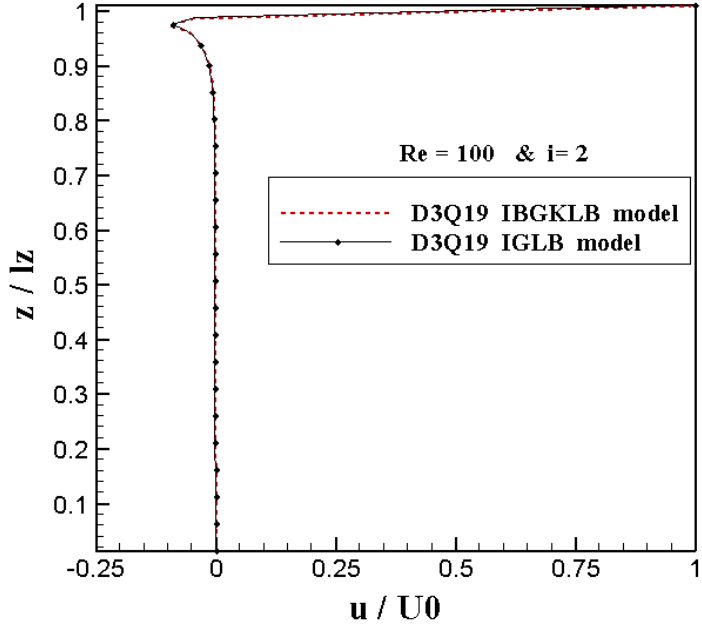

Fig. 13. Z-component velocity profiles at $i=2, R e=$ 100.

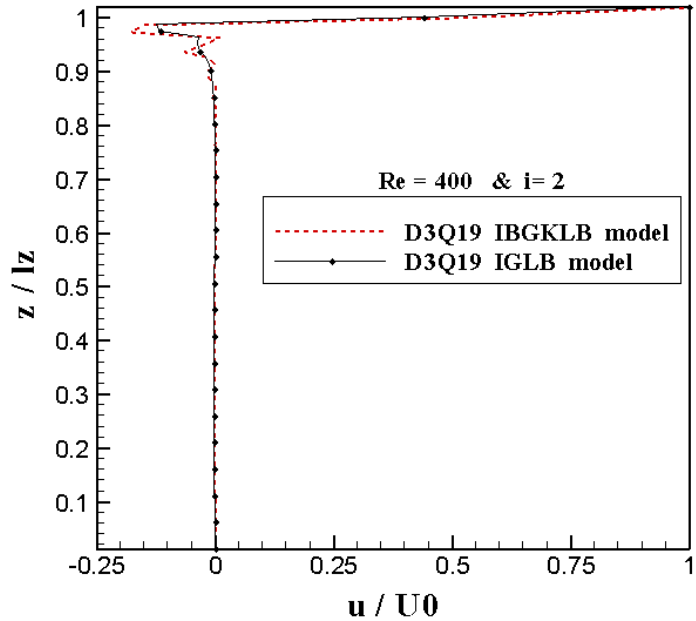

Fig. 15. Z-component velocity profiles at $i=2, R e=$ 400 .

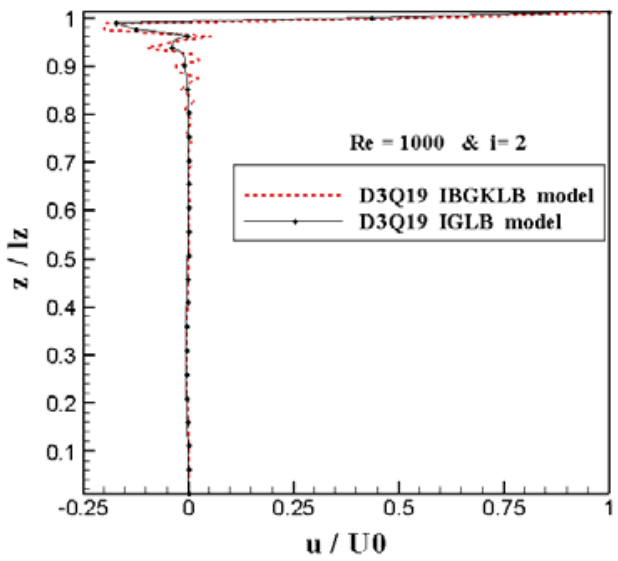

Fig. 17. Z-component velocity profiles at $i=2, R e=$ 1000. 
A. R. Rahmati and M. Ashrafizaadeh / JAFM, Vol. 2, No. 1, pp. 71-96, 2009.

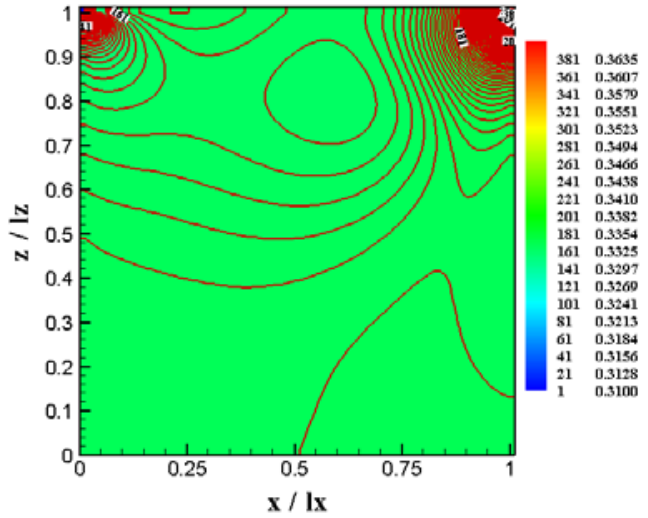

a) D3Q19-IBGKLB model

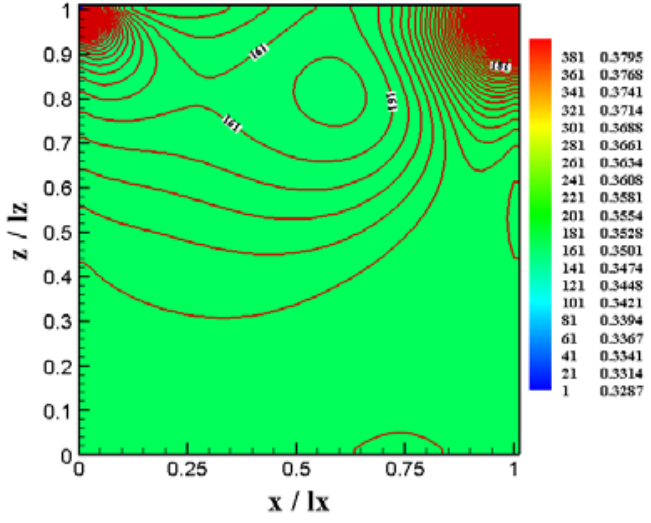

b) D3Q19-IGLB model

Fig. 18. Pressure contours at mid-plane of $Y=0.5$ for driven cavity flow at $R e=100$ using a) D3Q19-IBGKLB model and b) D3Q19-IGLB model with an $81 \times 81 \times 81$ lattice.

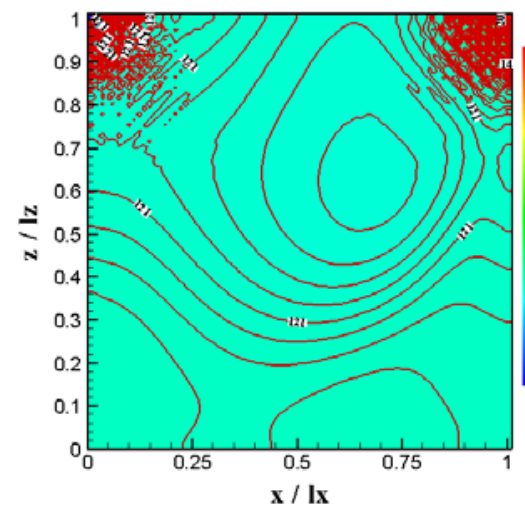

a) D3Q19-IBGKLB model

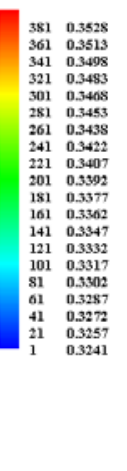
$Y=0.5$ for driven cavity flow at $R e=400$ using a) D3Q19-IBGKLB model

Fig. 19. Pressure contours at mid-plane of $Y=0.5$ for driven cavity
and b) D3Q19-IGLB model with an $81 \times 81 \times 81$ lattice.

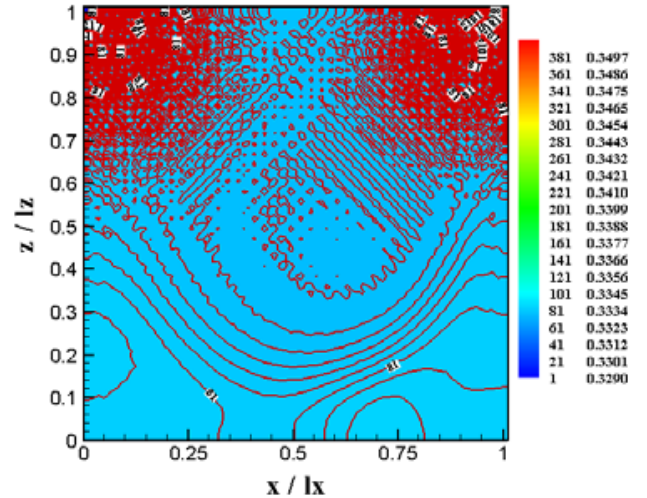

a) D3Q19-IBGKLB model

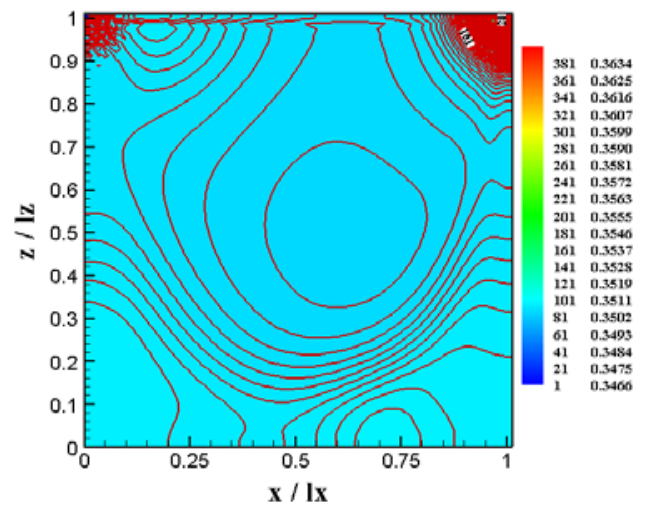

b) D3Q19-IGLB model

Fig. 20. Pressure contours at $Y=0.5$ for driven cavity flow at $R e=1000$ using a) D3Q19-IBGKLB model and b) D3Q19-IGLB model with an $81 \times 81 \times 81$ lattice. 
A. R. Rahmati and M. Ashrafizaadeh / JAFM, Vol. 2, No. 1, pp. 71-96, 2009.

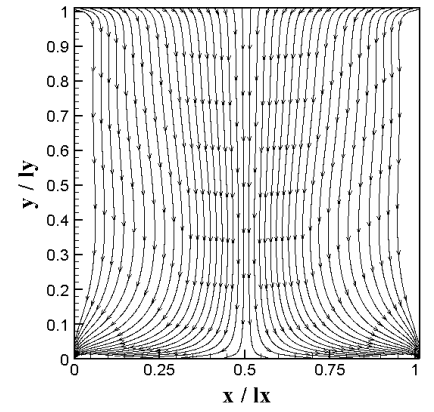

(a) $Z=0.5$

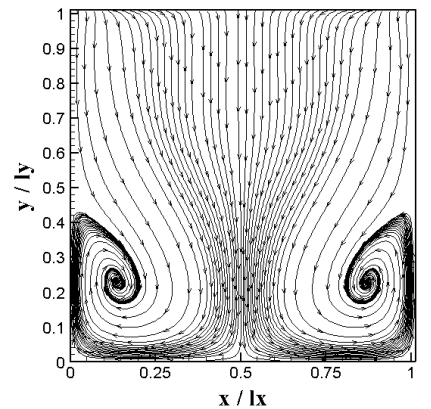

(a) $Z=0.5$

Continue ...

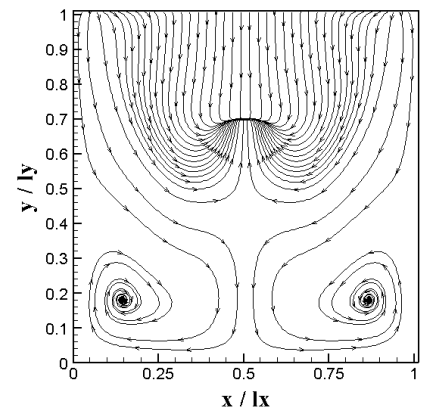

(a) $Z=0.5$

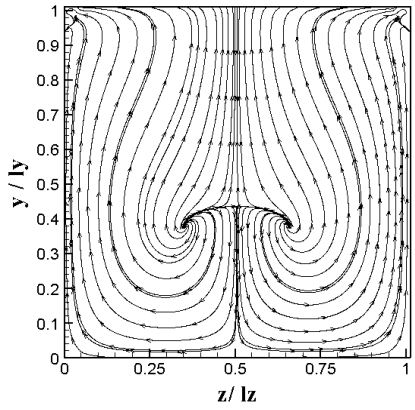

(b) $X=0.5$

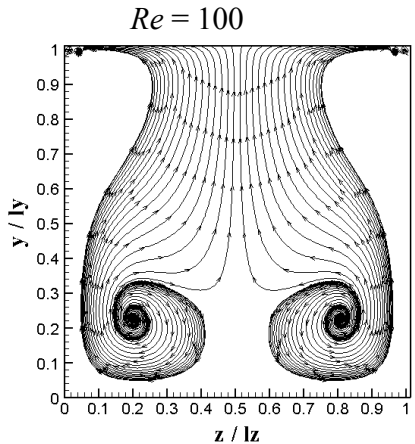

(b) $X=0.5$

$R e=400$

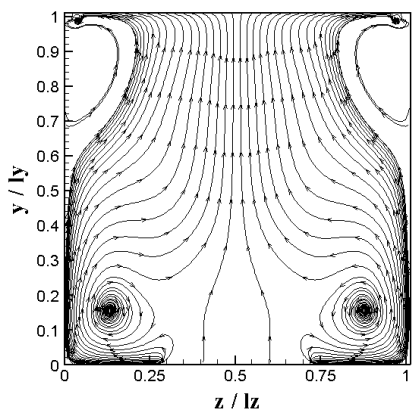

(b) $X=0.5$

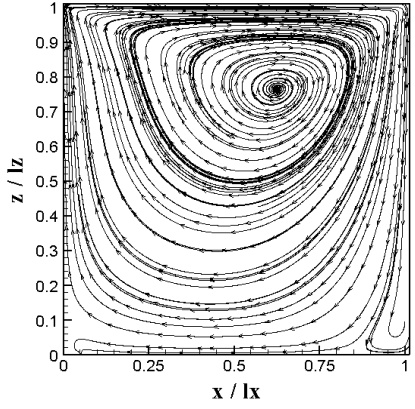

(c) $Y=0.5$

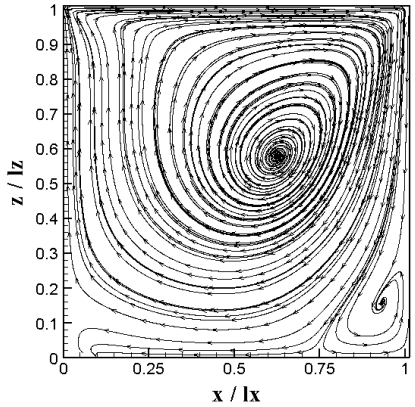

(c) $Y=0.5$

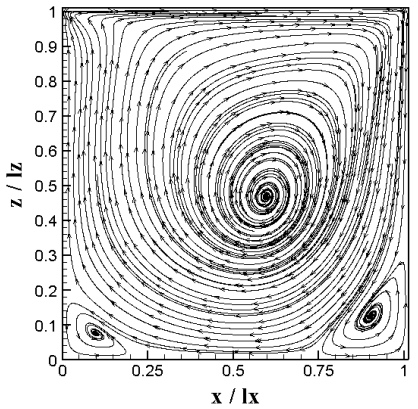

(c) $Y=0.5$

Fig. 21. Streamlines at different $R e$ using D3Q19 IGLB model and an $81 \times 81 \times 81$ lattice.

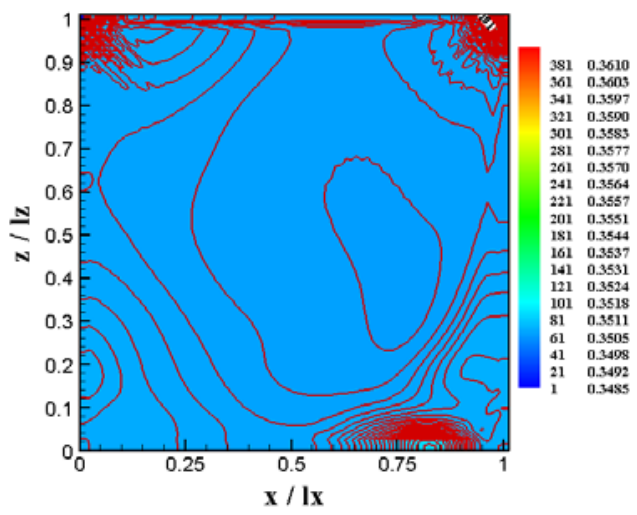

Fig. 22. Pressure contours at different planes for $R e=4000$ at 225000-th time step using D3Q19 IGLB model and an $81 \times 81 \times 81$ lattice. 
A. R. Rahmati and M. Ashrafizaadeh / JAFM, Vol. 2, No. 1, pp. 71-96, 2009.

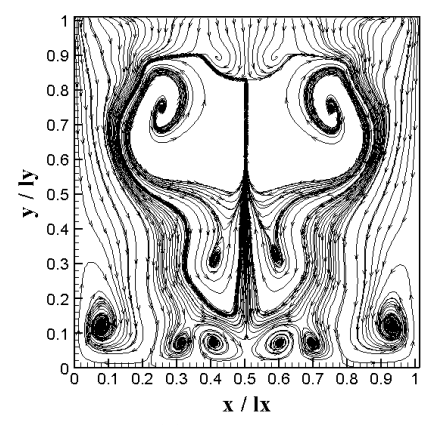

(a) $Z=0.5$

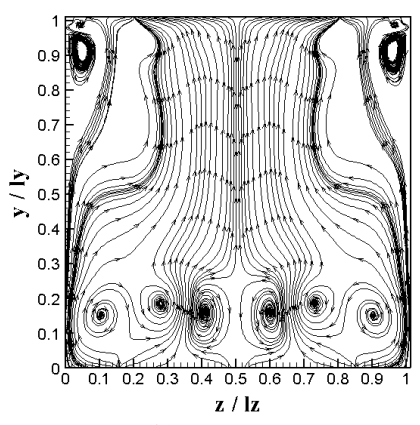

(b) $X=0.5$

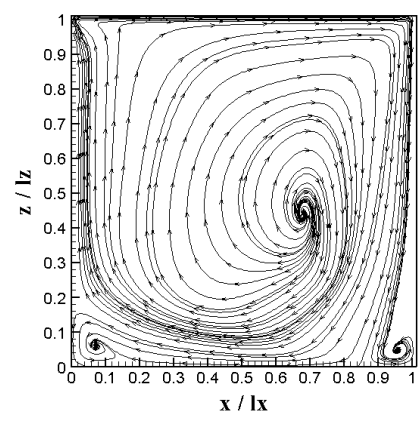

(c) $Y=0.5$

Fig. 23. Streamlines at different planes for $R e=4000$ at 225000 -th time step using D3Q19 IGLB model and an $81 \times 81 \times 81$ lattice.

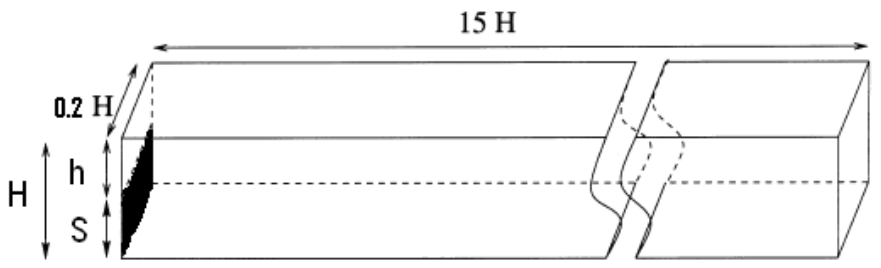

Fig. 24. Geometry of the backward-facing step channel. Flow is from left to right.

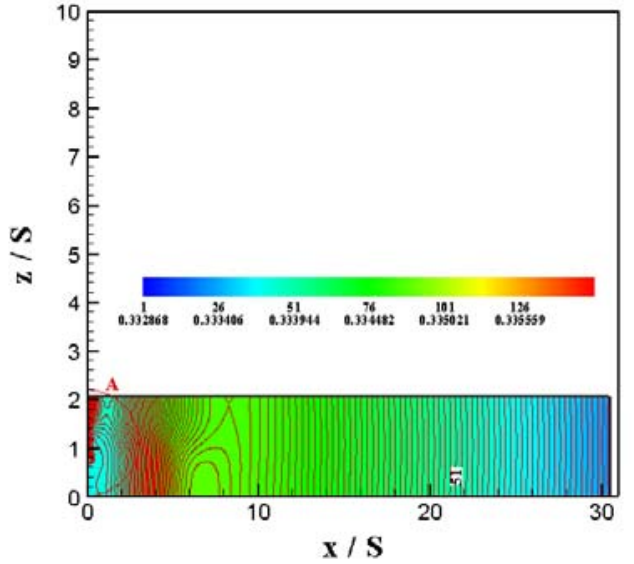

Fig. 25. Pressure contours at $Y=0.5$ for backward-facing step flow for $R e=200$ using D3Q19 IBGKLB model and a $945 \times 13 \times 63$ lattice.

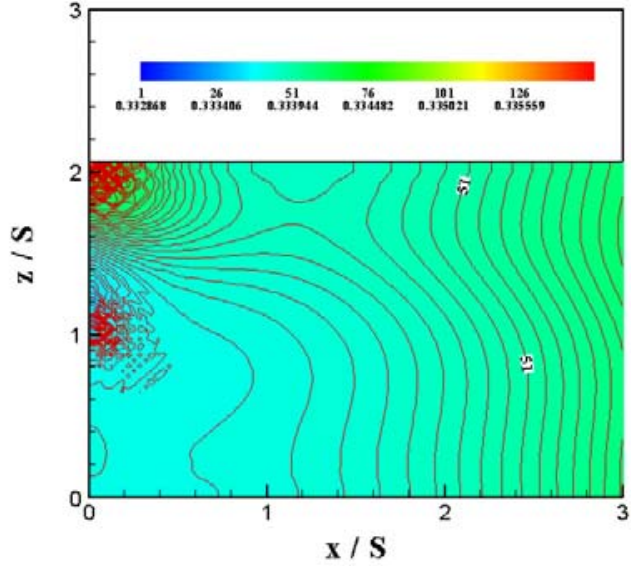

Fig. 26. Enlarged region A in Fig. 25. 
A. R. Rahmati and M. Ashrafizaadeh / JAFM, Vol. 2, No. 1, pp. 71-96, 2009.

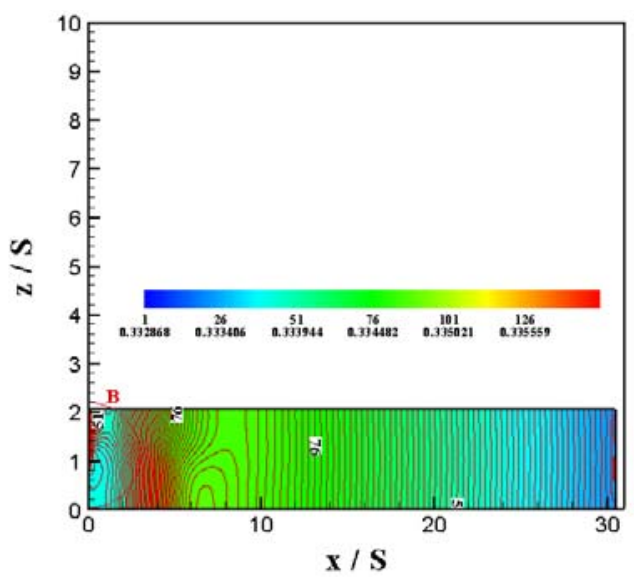

Fig. 27. Pressure contours at $Y=0.5$ for backward-facing step flow for $R e=200$ using D3Q19 IGLB model and a $945 \times 13 \times 63$ lattice.

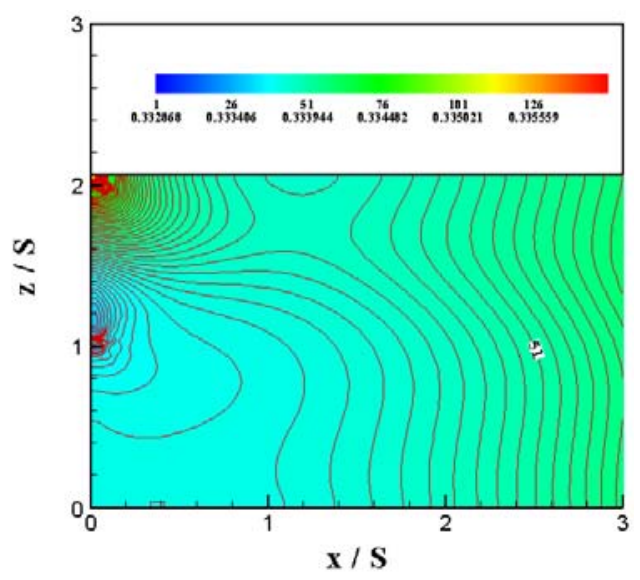

Fig. 28. Enlarged region B in Fig. 27.

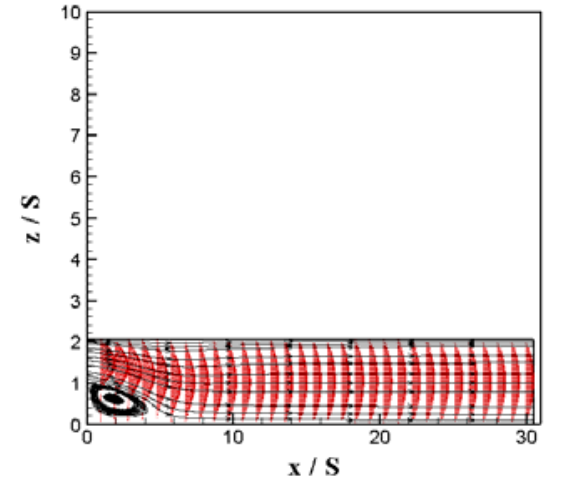

Fig. 29. Streamlines and velocity vectors at $Y=0.5$ for backward-facing step flow for $R e=200$ using D3Q19 IGLB model and a $945 \times 13 \times 63$ lattice.

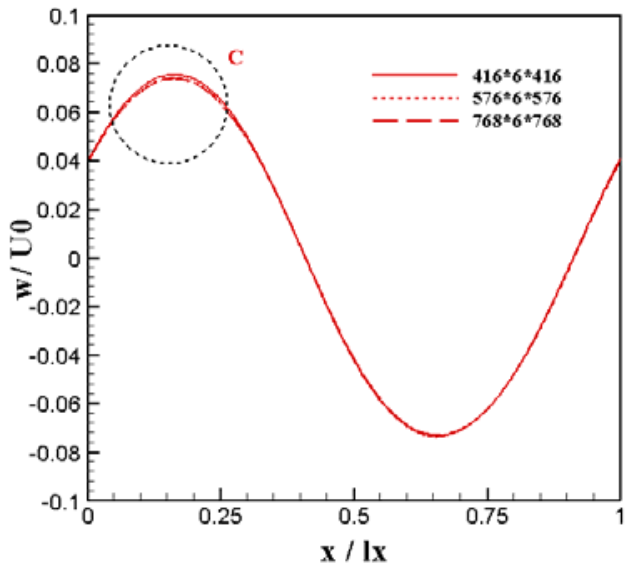

Fig. 30. Z-component velocity along the horizontal centerline of double shear flow for $t=0.8$ and different mesh sizes.

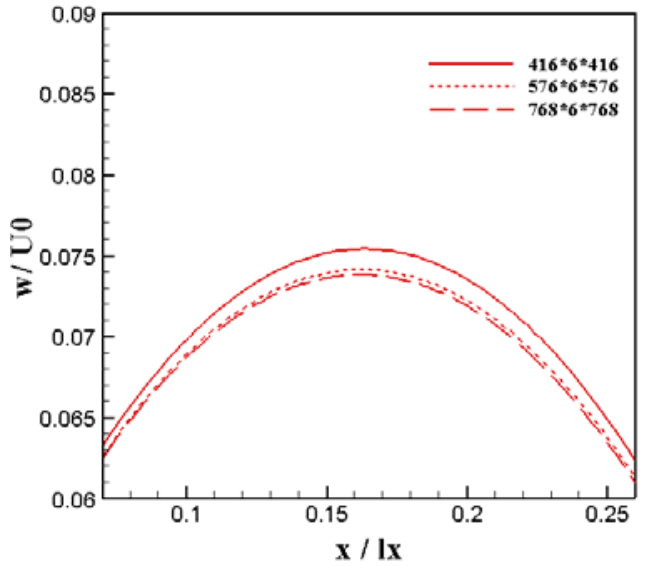

Fig. 31. Enlarged region C in Fig. 30. 
A. R. Rahmati and M. Ashrafizaadeh / JAFM, Vol. 2, No. 1, pp. 71-96, 2009.

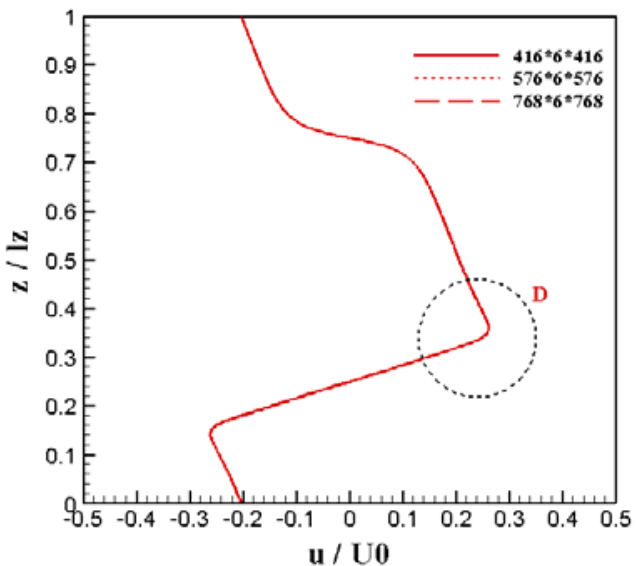

Fig. 32. X-component velocity along the vertical centerline of double shear flow for $t=0.8$ and different grid sizes.

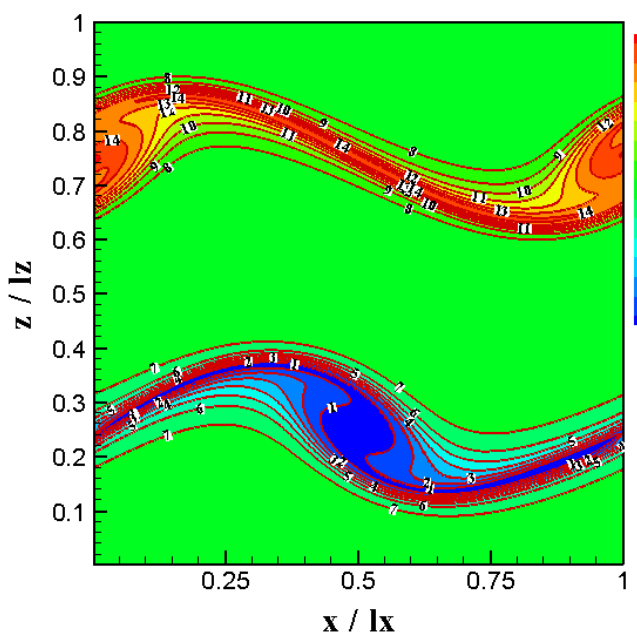

a) IGLB model

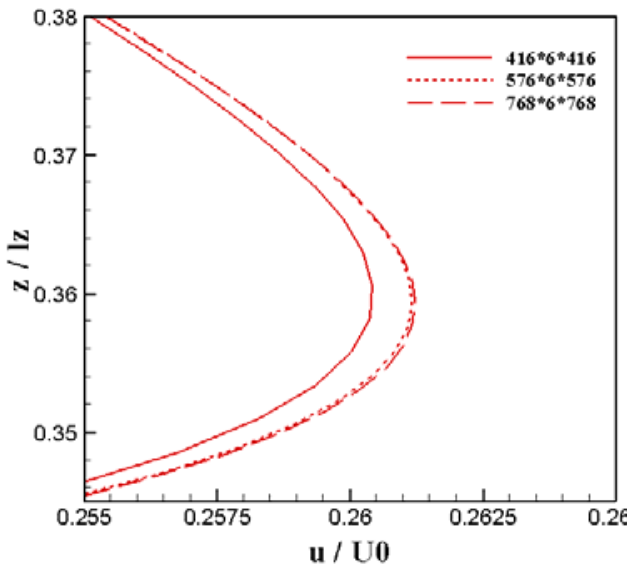

Fig. 33. Enlarged region D in Fig. 32.

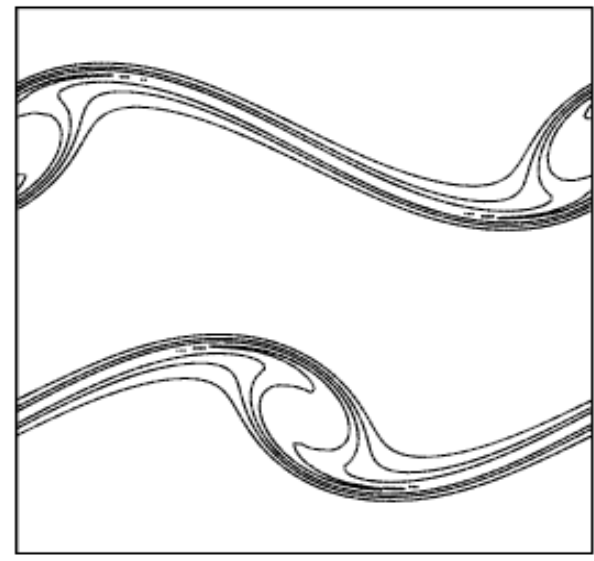

b) Moving mesh finite-element method by Di et al. (Di et al. 2005)

Fig. 34. Vorticity contours at $t=0.8$ with $k=30$ and viscosity $=1 / 10,000$ at $Y=0.5$ (all quantities are in lattice units).
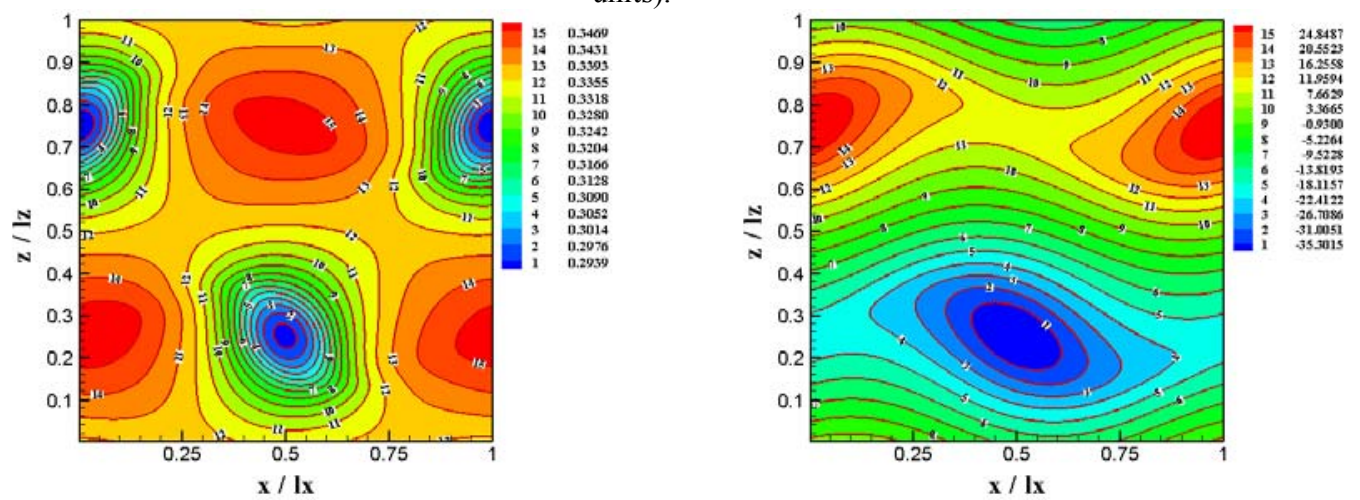

Fig. 35. Pressure contours (left side) and streamlines (right side) obtained by the IGLB model at $t=0.8$ with $k=$ 30 viscosity $=1 / 10,000$ at $Y=0.5$ (all quantities are in lattice units). 
A. R. Rahmati and M. Ashrafizaadeh / JAFM, Vol. 2, No. 1, pp. 71-96, 2009.

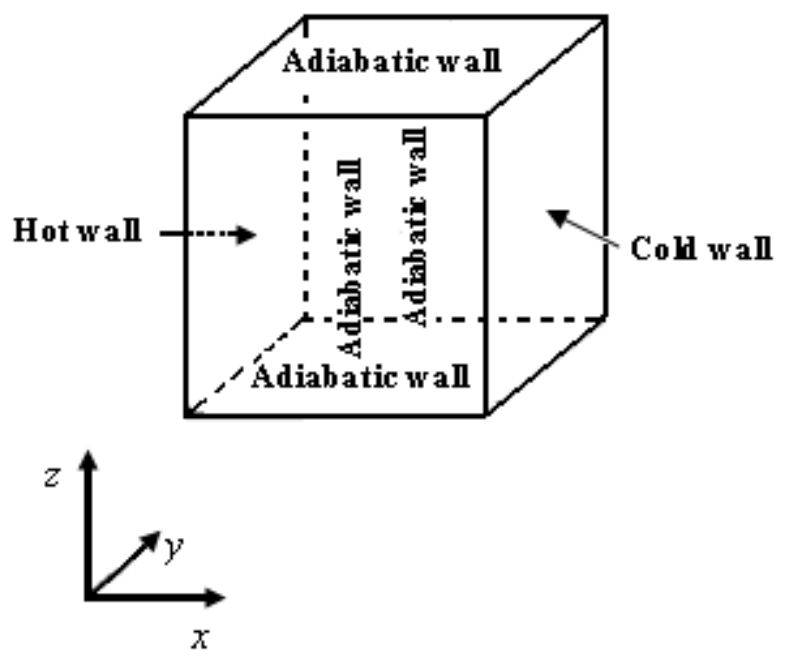

Fig. 36. Configuration of natural convection in a cubic cavity.

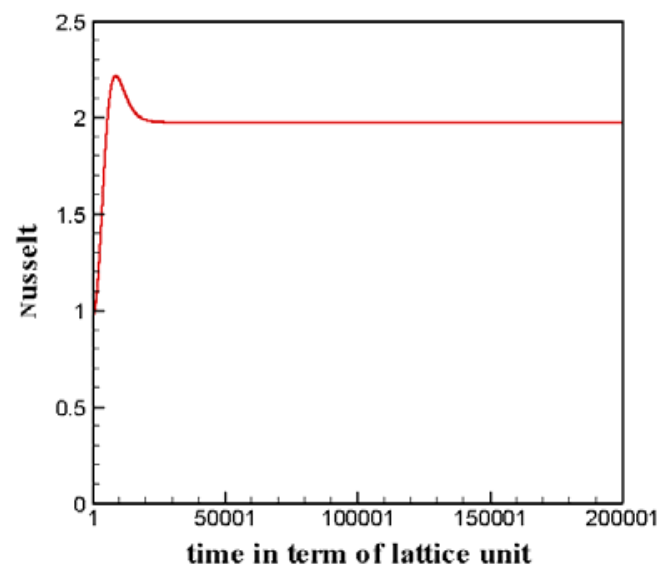

a) $R a=10^{4}$

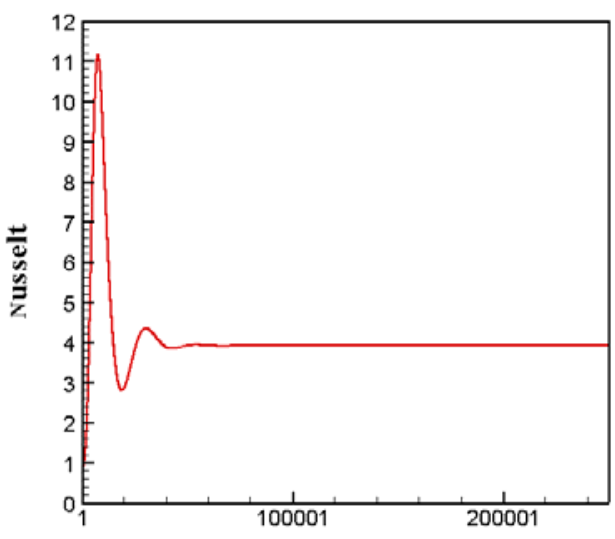

time in term of lattice unit

b) $R a=10^{5}$

Fig. 37. Time history of Nusselt number at different $R a$.

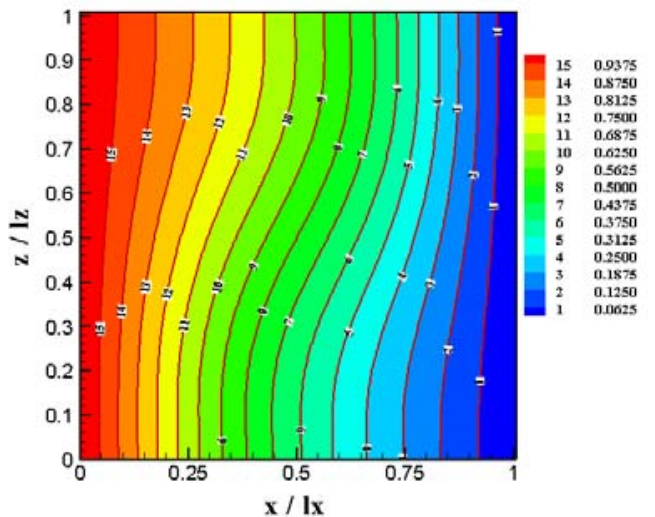

a) $R a=10^{3}$

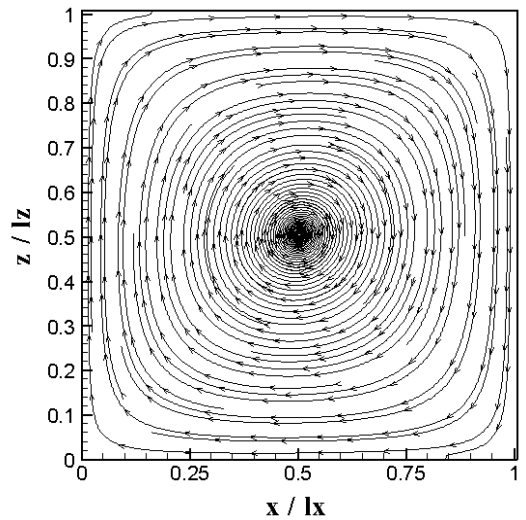

Continue ... 
A. R. Rahmati and M. Ashrafizaadeh / JAFM, Vol. 2, No. 1, pp. 71-96, 2009.
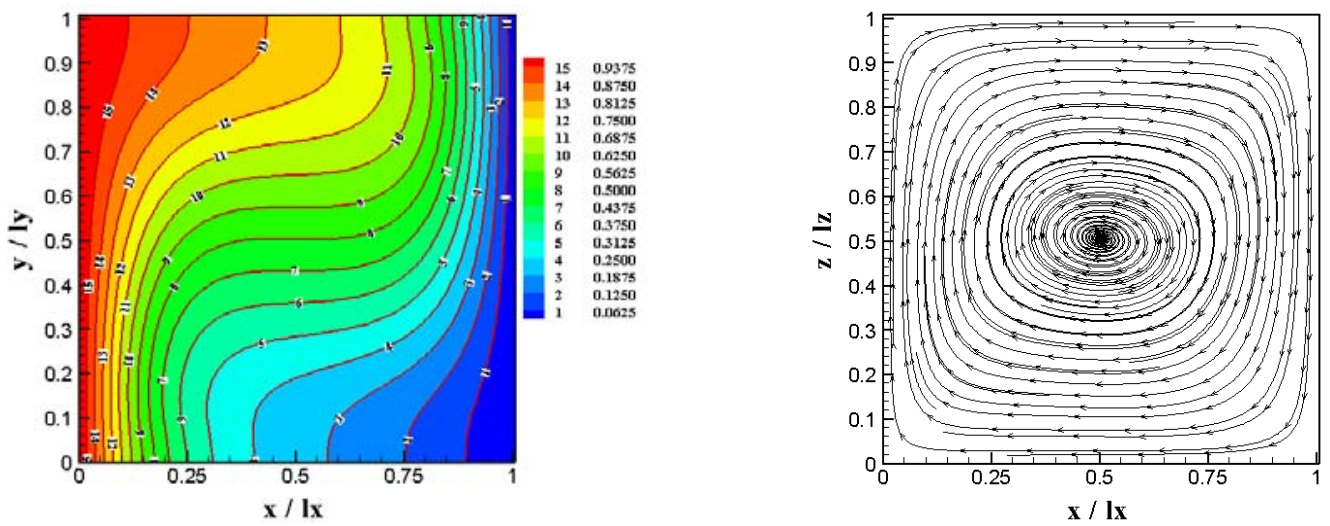

b) $R a=10^{4}$
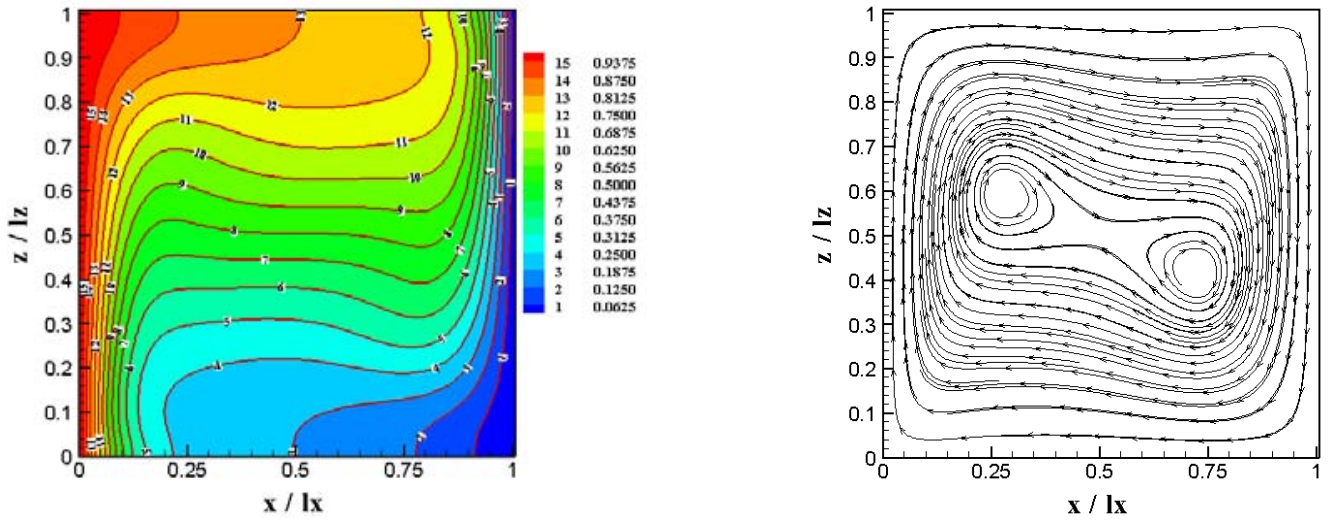

c) $R a=10^{5}$
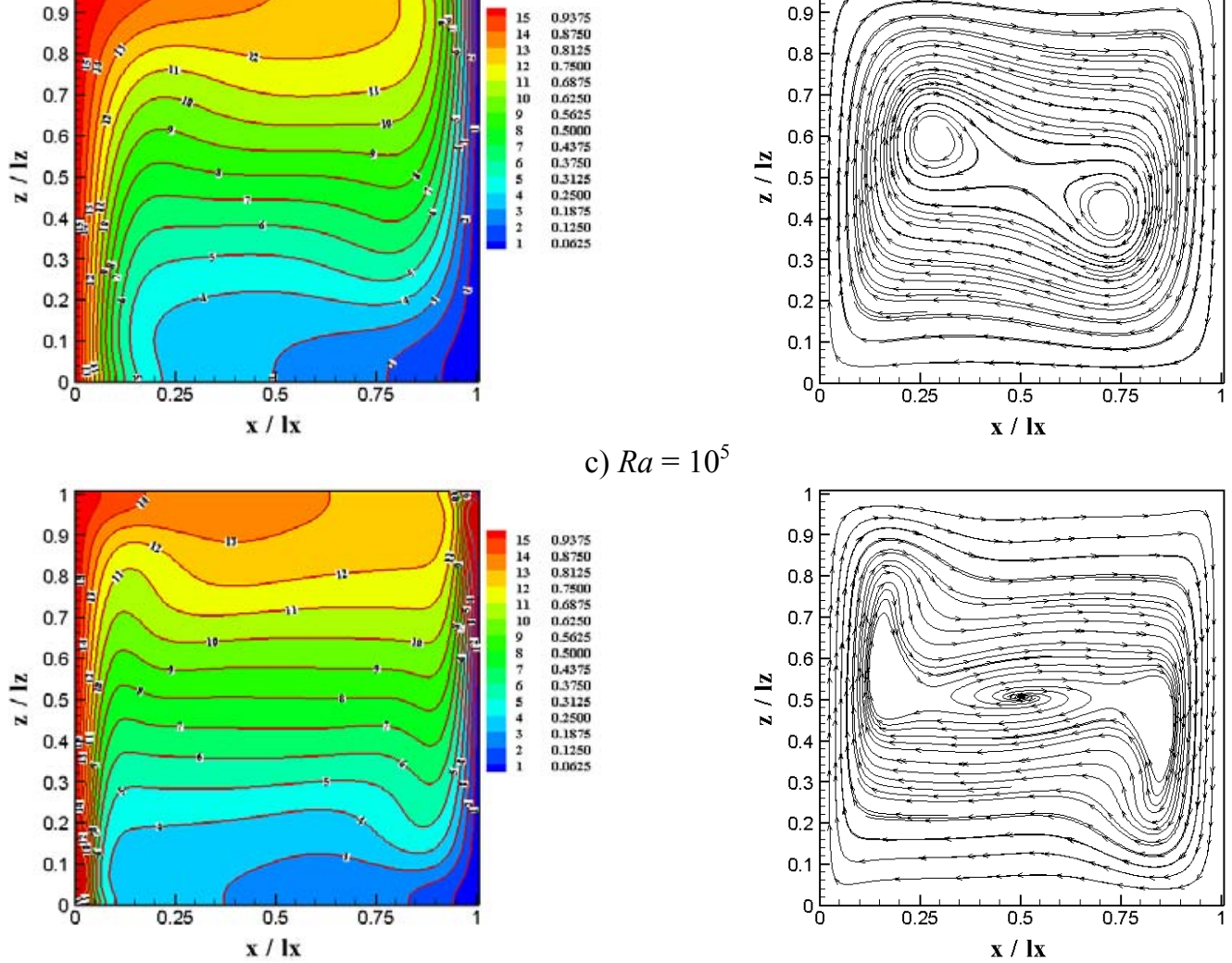

d) $R a=10^{6}$

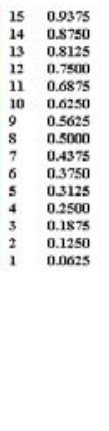

Fig. 38. Temperature contours (left) and streamlines (right) for the cubic differentially heated buoyant cavity flow for steady states at different $R a$ at middle plane of $Y=0.5$. 


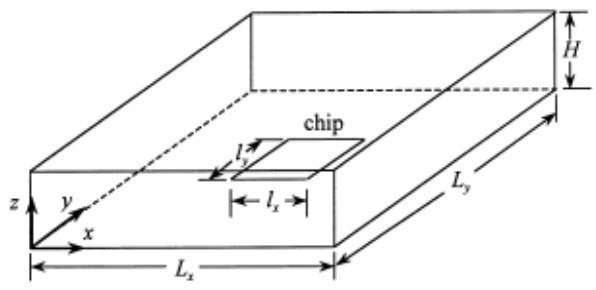

Fig. 39. Physical configuration and coordinate system used by Sezai and Mohamad (2000).

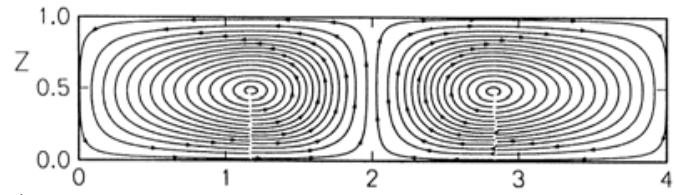

a)

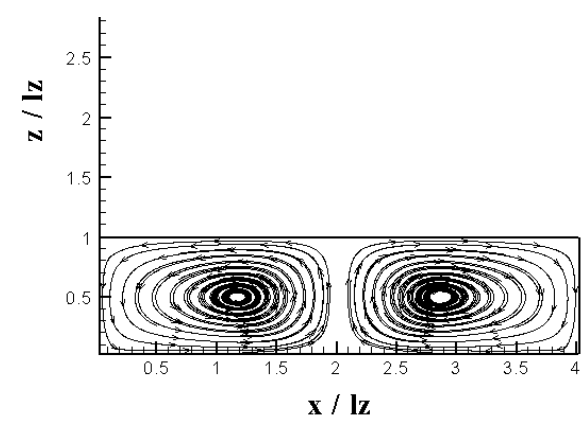

b)

Fig. 40. Projection of streamlines (left) and isotherms (right) of a) Sezai and Mohamad (2000) and b) the present work on the mid-plane of $Y=0.5$ for a discrete heater for $R a=1000$.

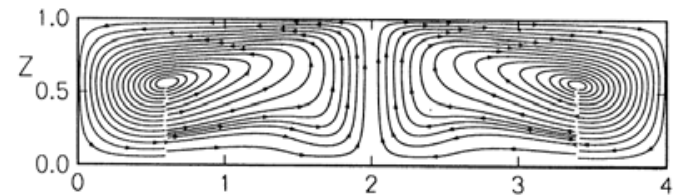

a)

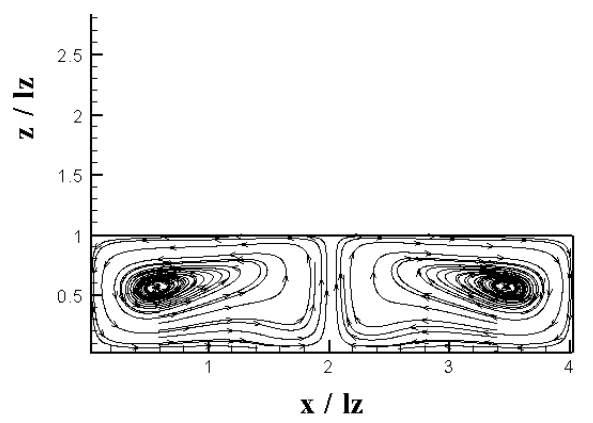

b)

Fig. 41. Projection of streamlines (left) and isotherms (right) of a) Sezai and Mohamad (2000) and b) the present work on the mid-plane of $Y=0.5$ for a discrete heater for $R a=10^{5}$. 